\title{
The Dawn Topography Investigation
}

\author{
C.A. Raymond - R. Jaumann - A. Nathues - H. Sierks • T. Roatsch • F. Preusker • \\ F. Scholten · R.W. Gaskell • L. Jorda • H.-U. Keller • M.T. Zuber • D.E. Smith • \\ N. Mastrodemos $\cdot$ S. Mottola
}

Received: 24 September 2011 / Accepted: 14 November 2011 / Published online: 15 December 2011

(C) Springer Science+Business Media B.V. 2011

\begin{abstract}
The objective of the Dawn topography investigation is to derive the detailed shapes of 4 Vesta and 1 Ceres in order to create orthorectified image mosaics for geologic interpretation, as well as to study the asteroids' landforms, interior structure, and the processes that have modified their surfaces over geologic time. In this paper we describe our approaches for producing shape models, plans for acquiring the needed image data for Vesta, and the results of a numerical simulation of the Vesta mapping campaign that quantify the expected accuracy of our results. Multi-angle images obtained by Dawn's framing camera
\end{abstract}

Government sponsorship acknowledged.

C.A. Raymond $(\bowtie) \cdot$ N. Mastrodemos

Jet Propulsion Laboratory, California Institute of Technology, Pasadena, CA 91109-8099, USA

e-mail: carol.a.raymond@jpl.nasa.gov

R. Jaumann · T. Roatsch · F. Preusker $\cdot$ F. Scholten $\cdot$ S. Mottola

Institute of Planetary Research, Deutsches Zentrum für Luft- und Raumfahrt (DLR), Berlin, Germany

A. Nathues · H. Sierks

Max-Planck-Institut für Sonnensystemforschung, Katlenburg-Lindau, Germany

R.W. Gaskell

Planetary Science Institute, Tucson, AZ, USA

L. Jorda

Laboratoire d'Astrophysique de Marseille, Marseille, France

H.-U. Keller

Dept. of Earth Sciences, Remote Sensing of the Earth and Planets, Freie Universität Berlin, Berlin, Germany

M.T. Zuber · D.E. Smith

Department of Earth, Atmospheric and Planetary Sciences, Massachusetts Institute of Technology, Cambridge, MA 02139-4307, USA

D.E. Smith

Solar System Exploration Division, NASA Goddard Space Flight Center, Greenbelt, MD 20771, USA 
will be used to create topographic models with $100 \mathrm{~m} /$ pixel horizontal resolution and $10 \mathrm{~m}$ height accuracy at Vesta, and $200 \mathrm{~m} /$ pixel horizontal resolution and $20 \mathrm{~m}$ height accuracy at Ceres. Two different techniques, stereophotogrammetry and stereophotoclinometry, are employed to model the shape; these models will be merged with the asteroidal gravity fields obtained by Dawn to produce geodetically controlled topographic models for each body. The resulting digital topography models, together with the gravity data, will reveal the tectonic, volcanic and impact history of Vesta, and enable co-registration of data sets to determine Vesta's geologic history. At Ceres, the topography will likely reveal much about processes of surface modification as well as the internal structure and evolution of this dwarf planet.

Keywords Vesta $\cdot$ Ceres $\cdot$ Dawn $\cdot$ Asteroid topography

\section{Introduction}

The objectives of the Dawn mission are to investigate the processes and conditions at the earliest epoch of formation of our solar system. The mission achieves this objective by comprehensively mapping the two most massive asteroids in the main belt, protoplanets 4 Vesta and 1 Ceres. The shapes and detailed topography of these bodies record many of the processes that have defined collectively their history, including differentiation, volcanism, convective motions and impact bombardment, as well as the nature of the lithosphere through its modification over time. Vesta has a basaltic surface that retains a record of ancient volcanic activity (McCord et al. 1970; Binzel and Xu 1993; Thomas et al. 1997; Li et al. 2010; Reddy et al. 2010; Pieters et al. 2011). It is also expected to have retained a record of its bombardment history, as evidenced by the putative large crater near the south pole detected by ground-based telescopic observations and the Hubble Space Telescope (HST). In contrast, Ceres' surface, which is predicted to be a regolith-covered ice-rich mantle that is warm relative to similar icy moons of the outer solar system, is expected to have erased many signatures of ancient impacts. The reconstruction of the shape of each body, along with their gravity fields, will allow inferences on their internal structure, while the basins, crater shapes, lineaments, ridges, graben, and volcanic constructs will provide a framework for interpreting the images and spectral data to determine the geologic history of each body. Comparison of the formation and evolution of Vesta and Ceres provides a context and ground truth for understanding many other objects in the asteroid belt and understanding the diversity of objects that coalesced to form the terrestrial planets.

Detailed mapping of topography is central to understanding processes shaping the surfaces of planetary bodies. Image data provide the basis for extensive studies of the surface structure and morphology on local, regional, and global scales using the photogeologic information from the image, the topographic information from the derived digital topographic model, spectral information from color images, and spectral reflectance data. Knowledge of the magnitudes as well as the directions of slopes is needed to understand such quantities as the viscosities of lavas, and hence their possible compositions, the volumes of volcanic constructs, the depths of craters/depressions, and the heights of mountains. Direct topographical measurements are also needed to recognize whether multiphase deformation is present and the degree of erosion of the surface.

Determining physical surface properties also provides geologic context. Identification of geologic units in terms of structure, age and compositional heterogeneities depends on local relief and the directions of slopes. Relationships of different geologic units define stratigraphic sequences, which are used to model the processes that formed specific surfaces. 
Topographic measurements are crucial for these investigations and are also used to refine the geodetic control network needed for gravimetric investigations. A comprehensive evaluation of the measurements from the Dawn instruments, based on the image-derived topographic image base maps, will enable combined structural/compositional investigations using the framing camera and the visible and infrared mapping spectrometer (VIR) and gamma ray and neutron detector (GRaND) spectrometers. Finally, the photogrammetric analyses are essential to coregistering the diverse data sets at sufficient accuracy that they will be useful.

\section{Current Knowledge}

Shape models of both asteroids have been constructed using telescopic data; the most recent and best data are from HST (Thomas et al. 1997, 2005; Drummond and Christou 2008; Carry et al. 2008). A digital shape model of Vesta's topography has been constructed from the original HST images, that is referenced to an equipotential surface (or an ellipsoidal approximation of one), assuming Vesta to be internally homogeneous (Thomas et al. 1997). The largest topographic feature is a depression that appears near the south pole and averages $460 \mathrm{~km}$ in diameter, with an average depth below the rim of $13 \pm 3 \mathrm{~km}$. Thomas et al. (1997) interpret this feature as a single crater. Rim heights above the average surface outside this crater range from $4 \pm 3$ to $12 \pm 3 \mathrm{~km}$; the central peak is about $13 \pm 3 \mathrm{~km}$ above the deepest part of the floor. Several other depressions, interpreted as craters by Thomas et al. (1997), are a $160 \mathrm{~km}$ diameter feature, $6 \pm 3 \mathrm{~km}$ deep, located at $20^{\circ} \mathrm{N}, 70^{\circ} \mathrm{W}$ and another of $150 \mathrm{~km}$ across, $8 \pm 3 \mathrm{~km}$ deep, located at $10^{\circ} \mathrm{N}, 270^{\circ} \mathrm{W}$, which is also reported as geologic feature in spectroscopic ground-based data (Gaffey 1997). The overall relief of Vesta ranges from $-12 \mathrm{~km}$ to $+12 \mathrm{~km}$ and is consistent with other planetary topographies.

For Ceres, fewer data exist, but analysis of limb images by Thomas et al. (2005) has revealed the two axial diameters of Ceres to be 909 (polar) and $975 \mathrm{~km}$ (equatorial), whereas near-infrared imaging yields an estimate of $a=b=479.7 \pm 2.3 \mathrm{~km}, c=444.4 \pm 2.1 \mathrm{~km}$ for the principal axes of an oblate spheroid that describes Ceres' shape (Carry et al. 2008). These dimensions indicate Ceres has a relaxed shape, consistent with a rocky core, mantled by an ice-rich layer (Thomas et al. 2005), as is predicted by thermal evolution arguments (McCord and Sotin 2005; Castillo-Rogez and McCord 2010). Brightness variations on Ceres seen by HST could be relict craters defined by compositional variations ( $\mathrm{Li}$ et al. 2006), but thermal arguments indicate large scale topography is unlikely to be found on Ceres due to viscous creep of the putative ice mantle.

\section{Determining Asteroid Topography}

Dawn's original payload included a laser altimeter that was similar to that carried on MESSENGER (Cavanaugh et al. 2007), but this instrument had to be eliminated from the payload during development. The imaging data obtained with Dawn's framing camera (Sierks et al. 2011) then became the primary topography data set, and the observation plans had to be designed accordingly. While Dawn's original plans had called for only nadir imaging, the needs of the topographic mapping investigation dictated multi-angle imaging. Dawn's objective is to map Vesta over at least $80 \%$ of the surface with $10 \mathrm{~m}$ height accuracy at $<100$ $\mathrm{m} /$ pixel. For Ceres, the resolution is at least $20 \mathrm{~m}$ in height at $<200 \mathrm{~m} / \mathrm{pixel}$.

Two main approaches will be used to quantify the topography of the surface of Vesta and Ceres; these are stereophotogrammetry (Stereo) and stereophotoclinometry (SPC), also 
known as shape-from-shading. Both techniques are applied to multiple optically-observed images of the same region (Jaumann et al. 2007; Gaskell et al. 2008). Photogrammetry, the geometrical analysis of stereo pairs of optical images, has provided much of our knowledge about the quantitative topography of Earth and other planets and is a direct measurement of structural parameters. Photoclinometry is an alternative approach that can be applied to unpaired images and can achieve single-pixel resolution, because it is based on interpreting brightness (shading) variations in slopes rather than measuring geometrical parallaxes. Unlike Stereo, it depends on an assumed photometric function, and requires additional data for scaling and absolute referencing. SPC solves iteratively for the slopes, albedo, camera pointing, and position. The Dawn team will also use a refined photoclinometric technique (photoclinometry by deformation, or PCD) that can refine the photometric properties by minimizing the misfit between the SPC model and the observations, thus reducing the photometric model-dependence in the photoclinometric solution (Capanna et al. 2011). All of these techniques lack accuracy over large distances due to the absence of a precise geodetic reference frame.

At Vesta, Dawn's topographic model will be constructed using data from the polar Survey orbit at a mean planetocentric radius of $3000 \mathrm{~km}$, and the polar High-Altitude Mapping Orbits (HAMO-1 and HAMO-2) at mean radii of $950 \mathrm{~km}$. Data from the Low-altitude Mapping Orbit (LAMO, $460 \mathrm{~km}$ radius) will also be used when appropriate. Dawn's imaging plans include $\sim 500$ images in Survey orbit, at $\sim 250 \mathrm{~m} /$ pixel resolution, each of which covers an area of $\sim 250 \mathrm{~km}^{2}$. The collection of Survey images will span a range of phase angles, including angles $<5$ degrees, and many images will include Vesta's limb. The main phase of multi-angle imaging occurs in the two HAMO phases; HAMO-1 occurs as a stop on the spiral into Vesta and HAMO-2 is timed to achieve the maximum change in illumination over the course of Dawn's stay at Vesta, during departure. In each HAMO sub-phase, multiple complete global mappings with overlapping images are planned. For HAMO-1, two nadir and four off-nadir acquisitions are planned, with a minimum phase angle of 30 degrees, and for HAMO-2, one nadir and three off-nadir mappings at 45 degrees minimum phase are obtained. Over 2500 clear filter images will be collected in HAMO-1 with a resolution of $\sim 70 \mathrm{~m} /$ pixel, and more that 1600 images in HAMO-2. More details of the observation plans are provided later.

\section{Analysis Methods and Validation}

Detailed descriptions of each technique (Stereo, SPC, and PCD) are provided in the following sections and the results of a validation exercise deemed "Virtual Vesta" is discussed. Finally, the process that will be followed to develop a final digital topographic model of Vesta is described. The method ties the topography to Vesta's center-of-mass, by simultaneously minimizing the misfit of surface features, gravity harmonics, and orbit crossover errors.

\subsection{Stereophotogrammetric Mapping (Stereo)}

Stereo data processing comprises two tasks that are described in detail below: (1) systematic processing of the raw data, and (2) photogrammetric processing for the derivation of three-dimensional models of the asteroid surfaces and ortho-rectified images (orthoimages). These tasks are performed iteratively until a full-resolution model is obtained. The raw FC data received from the spacecraft are sorted, adjusted by acquisition times, decompressed 
and radiometrically calibrated. Camera ancillary data (geometric distortion model), as well as other external geometric information such as orbit and attitude data, are available in the SPICE kernel format (http://naif.jpl.nasa.gov) that is used by almost all planetary missions. A variety of panchromatic and color three-dimensional products can be achieved by means of stereophotogrammetric processing, i.e. digital shape models (DSMs), multi-spectral orthoimages, image mosaics, and finally various types of 3D-visualizations.

DSMs are the main high-level products that serve as a precise description of Vesta's topography for science and for ortho-rectification of image data at high horizontal resolution. Considerable experience related to the photogrammetric processing of planetary data has been gained over more than 15 years from various missions, such as Mars Express (Jaumann et al. 2007), Lunar Reconnaissance Orbiter (Oberst et al. 2009; Scholten et al. 2009), MESSENGER (Preusker et al. 2011), Cassini and Galileo Missions (Giese et al. 2007), as well as using an airborne version of the HRSC sensor system (Wewel et al. 2000; Scholten and Gwinner 2004).

The stereophotogrammetric derivation of a DSM relies on area-based multi-image matching of two or more images. From the combination of the resulting tie point coordinates, combined with the camera calibration, and information of the orbit position and pointing, a 3-dimensional forward ray intersection yields an irregular grid of object points, which is used to interpolate a final raster DSM. Generally, the quality of DSMs and orthoimages strongly depends on the accuracy of the orientation data. While the interior camera geometry of the framing camera is well defined by laboratory calibration measurements, Dawn's spacecraft pointing data consists of reconstructed position and attitude based on radio tracking and optical navigation, which is combined with results from photogrammetric block adjustment information.

The photogrammetric DSM generation process chain (Fig. 1) is embedded in an iterative procedure, where reconstructed orbit and pointing data provided in SPICE format serve as initial input. A coarse-to-fine iteration includes photogrammetric block adjustment in order to improve the initial spacecraft/position, pointing direction, and the alignment of the FC camera frame to the Dawn spacecraft frame. Subsequently, intermediate DSMs are calculated and serve as input for pre-rectification within the respective iteration. When iterations for all stereo models of a global image dataset have yielded full-resolution tie point coordinates, an overall photogrammetric adjustment of the entire global block is used to derive the final orientation, which is used for the calculation of the entire global object point dataset and the final stereo DSM interpolation.

Pre-rectification Based on given orbit and pointing information the stereo observations are pre-rectified to a constant pixel scale in a common map projection. Thus, all stereo data sets are fitted to a common geometry. Additional a priori knowledge of the topography is used to reduce search areas for multi-image matching. Remaining image parallaxes reflect the deviation of the a priori topography assumption to the true shape of the body. In order to link this pre-rectification geometry to the raw image geometry, the raw image coordinates for each pre-rectified pixel are stored.

Multi-image Matching A multi-image matching technique is applied to the pre-rectified image data in order to derive conjugate points in each of the stereo observations. The algorithm makes use of area-based correlation in image pyramids to derive approximate values for the image coordinates, which are refined to sub-pixel accuracy by least-squares matching (Gwinner et al. 2009a). The correlation is done for each image as the reference image with all stereo partners, i.e. all overlapping images. A pyramidal approach using stepwise 
Fig. 1 Processing flow for DSM generation from stereo data. The top tier of the flow is a coarse-to-fine iteration photogrammetric block adjustments

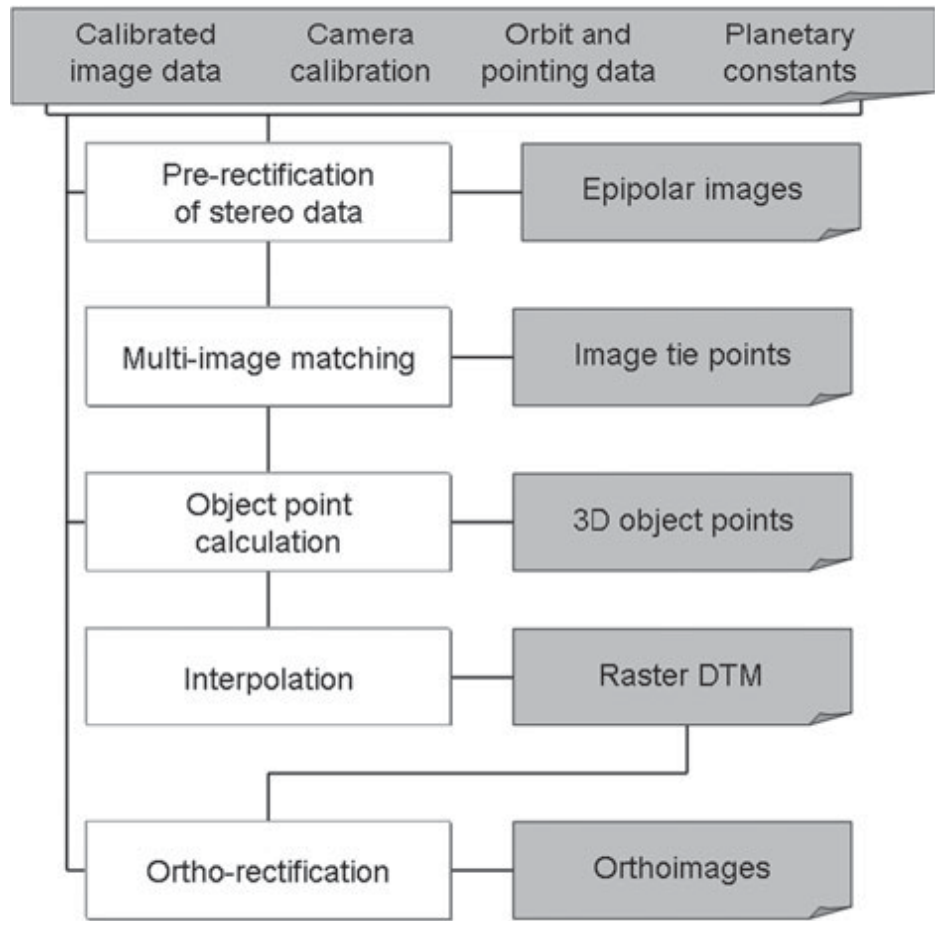

reduction of the correlation patch size yields moderate computation times and completeness even with large parallaxes. After the multi-image matching process, the derived image coordinates of all stereo observations are transformed back to raw radiometrically corrected data geometry, using the history files generated during the pre-rectification. The accuracy of this back-transformation is better than one tenth of a pixel (Scholten et al. 2005).

Object Point Calculation The lines of sight, defined by the image coordinate pair for each stereo observation, the geometric calibration, and the orientation data, are used for forward ray intersection. Least-squares adjustment is applied for this over-determined problem. The result is an object point and its relative accuracy defined in body-fixed Cartesian coordinates. The redundancy given by multi-stereo capability allows us to accept only those object points that are defined by at least three stereo observations. Thus, the influence of occasional gross matching errors, which cannot be avoided by simple two-image matching, is minimized.

Global DSM Interpolation For the generation of a DSM with a regular grid in a given map projection, object points from single stereo models as well as from a set of stereo models can be used. For the derivation of global DSMs, typically a large set of different models is combined to one, as will be done for derivation of global Vesta and Ceres DSMs. Object points are first transformed from body-fixed Cartesian coordinates to planetographic latitude/longitude referenced to a sphere, and height values for Vesta are based on either a 3 -axial ellipsoid with $a$-axis $=289 \mathrm{~km}, b$-axis $=280 \mathrm{~km}, c$-axis $=229 \mathrm{~km}$, or a sphere with $a$-axis $=b$-axis $=c$-axis $=270 \mathrm{~km}$ for easy data exchange. The planetographic latitude/longitude coordinates of all object points from all stereo image sets are transformed to standard map projections (Equidistant projection for equatorial regions up to $85^{\circ}$ Northern and Southern latitude, Stereographic projection for polar regions). Object points located within a DSM pixel are averaged, and can optionally be combined by different filtering techniques with those within a chosen neighborhood (Gwinner et al. 2009b). For regions that lack any object point information, a final gap-filling algorithm using DSM pyramids with reduced resolution can be applied in order to derive a raster DSM without gaps. 
Ortho-rectification and Mosaicking Each image can be ortho-rectified precisely using finally adjusted orientation data and shape as represented by the DSM. The ortho-rectification consists of ray-tracing regular pixel positions of the input radiometrically-corrected pixel matrix with the DSM using interior camera calibration and exterior orientation data related to the respective pixel (Scholten et al. 2005). For each grid point, a 3-D intersection point with the DSM is derived and transformed to the map projection of the output orthoimage (same standard projections as for the DSM). For each pixel within a mesh of these transformed grid points, an indirect resampling based on different selectable interpolation methods is performed within the radiometrically corrected input matrix in order to derive the final orthoimage grey value. Finally, ortho-rectified color and panchromatic channels, if rectified to the same map projection and pixel scale, fit to each other with the accuracy provided by the DSM and the applied orientation data and can be superimposed as co-registered multi-spectral/multi-phase data sets (Scholten et al. 2005). Ortho-rectified images of different Dawn orbits need to be combined for mapping of target areas spread over different orbits. For this purpose, a radiometric mosaicking tool has been developed. It combines all input orthoimages by simply copying them to one common image mosaic, and also allows brightness adjustment. No geometrical adjustment is applied; the geometric relationship between the images to be mosaicked is considered to be fixed and is defined by each image's label information comprising nominal map projection parameters, scale, and the offset of its upper left pixel with respect to the projection center.

Quality Assessment of $3 D$ Products Detailed quantitative statements about the data quality, the accuracy of the entire data processing line, and the derived products require intensive studies and statistical analyses, since the geometrical conditions of each orbit may vary (Scholten et al. 2005). Orbit maneuvers do not allow for a consistent quality of orientation data, but these are not expected in Survey and HAMO. The impact of variable local texture and varying illumination conditions are also difficult to include in an overall quality assessment.

Data Products and Image Maps Local, regional, and global DSMs, global clear filter mosaics, and global color mosaics will be produced. The Vesta topographic atlas will be produced at a scale of 1:250,000 or 1:500,000 and will consist of 15 tiles that conform to the quadrangle scheme proposed by Greeley and Batson (1990), for medium-sized planetary bodies. This tiling schema is described in Russell and Raymond (2011) and was recently used for the saturnian satellites like Enceladus (Roatsch et al. 2008).

\subsection{Stereophotoclinometric Mapping (SPC)}

Stereophotoclinometry is the process by which ortho-rectified projections of many images of a surface patch, ideally acquired under differing illumination conditions, are used to construct a landmark template called a maplet. These maplets can be used to identify the surface patch and locate its center in any image under any illumination or viewing geometry. The slope and albedo at each pixel of the maplet is determined by minimizing the brightness residuals between the images and the illuminated maplet in the least squares sense; shadows are treated as absent data. The derived slopes are then integrated to produce a digital shape model (DSM). A flowchart showing the inputs, software modules and outputs of the SPC method is shown in Fig. 2.

As Dawn approaches Vesta, an initial set of maplets will be constructed from the first images. As Vesta rotates, a given piece of surface is seen with varying local sun elevation, 


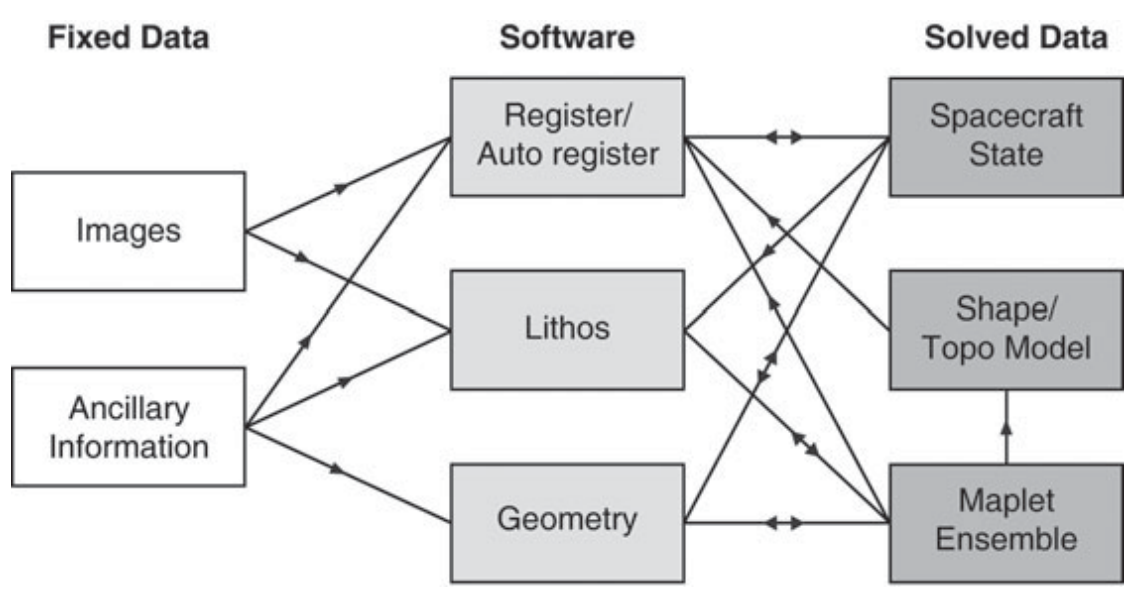

Fig. 2 SPC processing flow from inputs (left) through principal software functions (middle) to products $($ right $)$. The process is iterative within convergence limits

allowing the software module LITHOS to construct the topography that best describes the changes in the images caused by the changing illumination. A photometric model derived from Dawn observations and ground-based data will be used to initially define the albedo, which will be solved for along with the slopes, allowing refinement of the photometric model. Re-illuminated maplet images are correlated with the images themselves to determine the precise image space position of each maplet. Figure 3 shows a topography/albedo maplet constructed from 62 NEAR images of Eros, re-illuminated and compared with projected image data. The maplets display the major features of the images, and reproduce the brightness variations well. This information is fed into GEOMETRY, which determines the absolute location of the maplet on the body to a few tenths of a pixel.

By the start of Survey, several thousand maplets will have been constructed at a resolution comparable to the Survey images. These maplets will also have been combined to produce a detailed shape model of Vesta. As each new Survey image is obtained, it is first processed with REGISTER to correlate it with the illuminated shape model, refining the initial camera pointing. AUTOREGISTER then finds all maplets appearing in the image, re-illuminates them, determines where they are in the image and uses this information to update the spacecraft state estimate. Each maplet occurring in the new image is then processed with LITHOS, where the new image data refines its shape, albedo and location. During Survey, thousands of higher resolution maplets will be constructed, not only to refine the global shape but to be ready for the processing of HAMO images. By the time Dawn leaves Vesta, several hundred thousand maplets will have been constructed, at horizontal spatial resolutions as high as 20 meters. SPC has ultimately achieved height accuracies of one-tenth horizontal pixel resolution for previous modeling of bodies such as Eros and Itokawa. A summary of the results, including the starting and derived photometric model, are given in Gaskell et al. (2008).

The large number of images in which a maplet can be found makes it possible to determine its surface location very precisely, since spacecraft position errors and camera pointing uncertainties are averaged down. Similarly, a large number of maplets in a single image allows for the spacecraft position and camera pointing estimates to be refined. Figure 4 shows a display from the AUTOREGISTER program that solves for the spacecraft state by aligning a MESSENGER image with the ensemble of existing maplets. A maplet surface does not have to be completely within an image to be usable. 

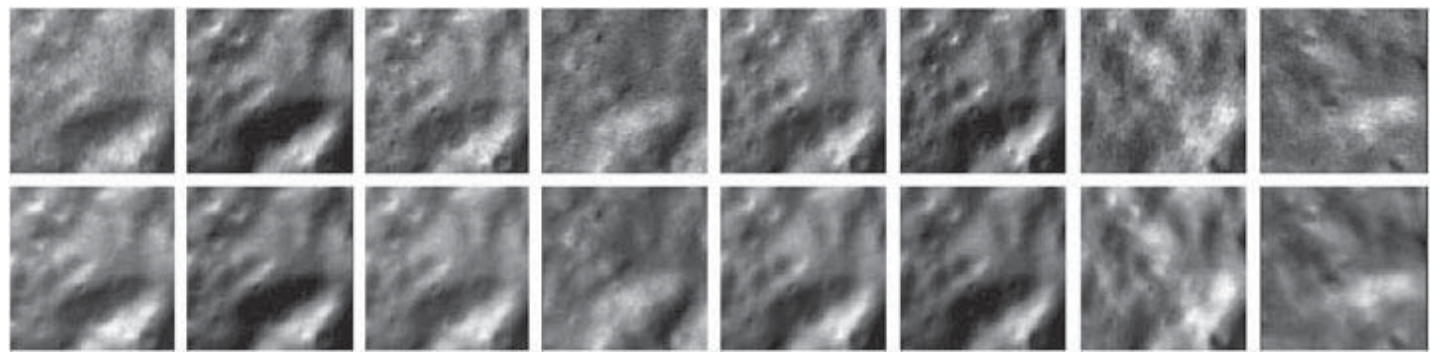

Fig. 3 Illuminated maplet (bottom row) and projected image data (top row)

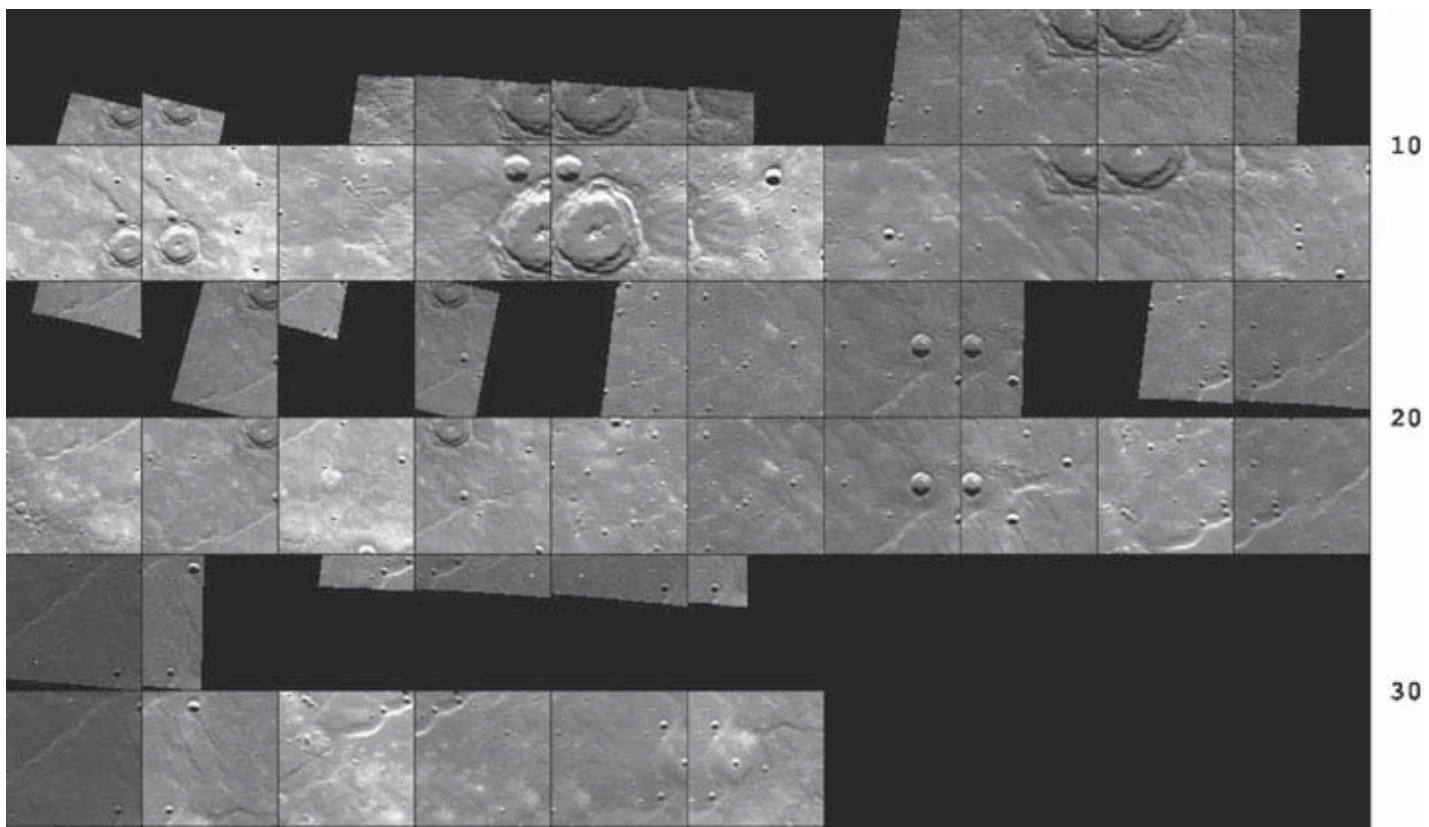

Fig. 4 An AUTOREGISTER display of projected MESSENGER Dual Imaging System (MDIS) data (Hawkins et al. 2001). As each new image is added, the maplets it contains (even rows) are illuminated and correlated with the extracted image data shown above them (odd rows). The image-space locations determined in this way quickly correct the camera pointing and s/c position solution

The maplet ensemble is more powerful than a collection of single maplets. If many maplets in an image have effectively registered it, other maplets appearing on the limb of the body in that image are more tightly constrained. If two maplets overlap one another, their common topography provides a three-dimensional constraint between the body-fixed locations of their centers. Finally, if the ensemble tiles the entire body then they can be combined to produce a global shape model for the body.

SPC processing has been used on small bodies such as asteroids Eros and Itokawa and large ones such as Mercury. Vesta is small enough to have more in common with the former. First, it is small enough that landmark tracking must play a significant role in the navigation strategy (Gaskell 2011). Because maplets can be located in images at any resolution, illumination and viewing geometry, they provide excellent navigation tie points and, in fact, that was their original purpose. Second, like Eros and Itokawa, it is likely that some images will have maplets that are at least partially occluded by other parts of the body. The software is designed to handle this contingency. 


\subsection{Photoclinometry by Deformation (PCD)}

An extension of the SPC technique is photoclinometry by deformation (Capanna et al. 2011). This is a technique that allows refinement of shape models ("maplets") derived using other techniques such as stereophotogrammetry and stereophotoclinometry, described in Sects. 4.1 and 4.2. The method is applied to a collection of DSMs, typically the SPC maplets produced after the Survey, HAMOs and LAMO phases. PCD requires as input the set of DSMs describing the surface of the body, and accurate geometric information derived by the navigation team and further refined with the above-mentioned SPC and Stereo techniques: the pole orientation, the S/C-object vector in the body-fixed frame and the rotation matrix from the body-fixed frame to the camera frame for each camera image. It also requires input "photometric parameters" allowing calculation of the bi-directional reflectance of the surface; an albedo map can be taken as input. For Vesta, the photometric model constructed from ground-based observations and Dawn data will be used as a starting model. The optimization of a given DSM is achieved iteratively in four steps described below. The free parameters of the methods are the heights of the vertices of the DSM above the surface, with the DSM being described as a triangular mesh ("plate model").

1. Synthetic sub-images of the DSM corresponding to a set of typically 10 to 30 observed FC images are generated.

2. A "penalty function" equal to the chi-square difference between observed and synthetic images is calculated. The partial derivatives of the penalty function with respect to the free parameters also need to be calculated.

3. This function and its partial derivatives are passed to a quasi-newton non-linear optimization algorithm called "Limited-memory Broyden-Fletcher-Golbfarb-Shanno" (L-BFGS), which then modifies the free parameters in order to minimize the penalty function.

4. The method goes back to step 1 until a convergence criterion is reached. This method can be applied to a set of DSMs. The "photometric parameters" can be re-calculated from this new set of DSMs and the PCD method can then be iterated.

Although it has been successfully applied to the shape model of asteroids Steins and to DSMs of the asteroid Lutetia observed by the OSIRIS cameras aboard ROSETTA (Capanna et al. 2011), PCD is a newly developed method still under very active development. On Dawn, PCD DSMs and derived photometric parameters will be compared to the products of the Stereo and SPC processing pipelines, and could be used as inputs to the geodetic DSM. The PCD DSMs will also help refine the contributions of brightness variations due to surface properties from those due to slopes.

\subsection{Deriving a Geodetically-Referenced DSM}

The several different models of Vesta shape and gravity (Konopliv et al. 2011) produced by the Dawn team will be integrated to produce a single combined and internally consistent model of Vesta's topography and gravity field. The various shape models will be combined using their covariance matrices and statistical estimates of their quality to produce a model that retains the more reliable and accurate aspects of the individual models, while benefitting from the strengths of the different approaches. In addition, the Dawn radiometric tracking data will be re-analyzed using the best gravity model, together with the shape models, for potential improvement of both the shape and gravity. The analysis will be conducted within the GEODYN geophysical estimation program (Rowlands et al. 1993; Pavlis et al. 2001) used at the Goddard Space Flight Center. GEODYN has the ability to process a variety of geodetic and geophysical data types, including control points 


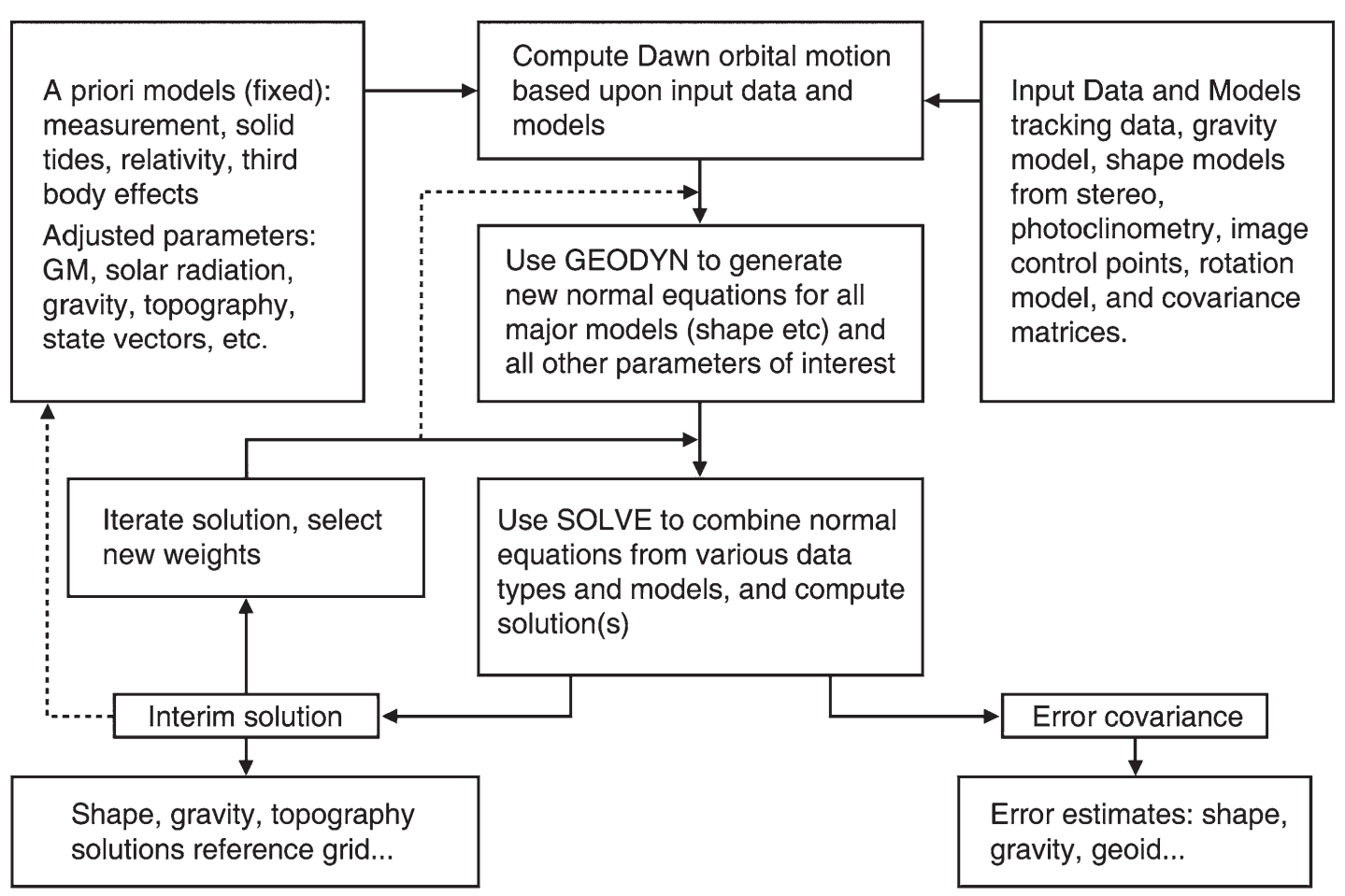

Fig. 5 Flowchart showing GEODYN methodology

from imaging data, and Doppler tracking data of various kinds (Zuber et al. 2000; Neumann et al. 2001). The program evaluates these data at orbit crossovers as well as in the normal orbit determination and gravity estimation process. A flowchart summarizing the GEODYN process is shown in Fig. 5. This combination of approaches and solutions will allow parameters to be derived using complementary techniques, thereby offering a potential improvement for the complete geodetic and geophysical model of Vesta. The primary objective of this approach is to obtain the best overall topography of Vesta relative to an equipotential surface, with a corresponding gravity field for interpretation of Vesta's interior and dynamics.

By the nature of the approach, most of this integrative work will be undertaken after the Dawn spacecraft has departed Vesta and begun its cruise to Ceres, after many of the primary models of Vesta topography and gravity have been developed.

\subsection{Observing Requirements for Image-Derived Topography}

The stereophotogrammetric method does not, apart from the generic requirement of overlapping images from different observation directions, depend on general imaging geometry. But quality and quantity of the DSM results are significantly affected by the viewing and illumination conditions involved. Based upon substantial experience from stereo processing of other planetary image data, observation and illumination requirements for Dawn's stereophotogrammetric processing have been defined in order to maximize the output quality. In a similar manner, conditions that contribute to better accuracy photoclinometricallyderived heights can be defined. Table 1 lists the optimal conditions for each technique, as well as the useable conditions that may not always produce the best height accuracy. 
Table 1 Optimal and useable parameter ranges for key observing attributes are given for both Stereo and SPC. The lower limit of the stereo angles is specified with two values indicating whether one pair within the set can have a lower minimum angle

\begin{tabular}{llllll}
\hline Parameters (in degrees) & Optimal & & & Useable & \\
\cline { 2 - 3 } & Stereo & SPC & & Stereo & SPC \\
\hline Illumination variation & $0-10$ & $20-70$ & $0-15$ & $0-70$ \\
Stereo angle & $15,15-55$ & $20,0-90$ & $15,10-65$ & $15,0-90$ \\
Incidence angle & $5-60$ & $10-75$ & & $0-80$ & $0-80$ \\
Emission angle & $0-70$ & $20-50$ & & $0-75$ & $0-70$ \\
\hline
\end{tabular}

For Stereo mapping, in priority order:

- The variation of the illumination direction of stereo images should be $<10^{\circ}$, to minimize mis-matching caused by variable illumination. Angles up to $15^{\circ}$ are useable.

- The angle between Stereo images should be $>15^{\circ}$ and $<55^{\circ}$, to provide sufficient vertical and horizontal accuracy. Angles as small as $10^{\circ}$ can be used.

- The incidence angle for each image segment should be $>5^{\circ}$ and $<60^{\circ}$, to provide sufficient contrast and to minimize the amount of cast shadow areas. Incidence as low as $0^{\circ}$ and as high as $80^{\circ}$ can be used depending on the surface characteristics.

- The emission angle for each image segment should be $<70^{\circ}$, to exclude near-limb image segments. Emission as high as $75^{\circ}$ can be used, and

- The phase angle for each image segment should be $>2^{\circ}$, to avoid influence from opposition effect.

For SPC, in priority order:

- The variation of the illumination direction of stereo images should be $>20^{\circ}$, to maximize the slope-dependent brightness variations. Angles down to $0^{\circ}$ are useable.

- The angles between three stereo images should be $>0^{\circ}$ and $<90^{\circ}$, with one angle being $>20^{\circ}$, to provide sufficient vertical and horizontal accuracy.

- The incidence angle for each image segment should be $>10^{\circ}$ and $<75^{\circ}$, to provide sufficient contrast and to minimize the amount of cast shadow areas. Incidence as low as $0^{\circ}$ and as high as $80^{\circ}$ can be used depending on the surface characteristics.

- The emission angle for each image segment should be $>20^{\circ}$ and $<50^{\circ}$. Emission as high as $70^{\circ}$ can be used, and

- The view angles should ideally be spread equally in $360^{\circ}$ around observation point

Considering these requirements, it is evident that the stereo and SPC techniques are complementary in exploiting different observing conditions, with some overlap. This is particularly well suited to Dawn, because our observing geometry is such that both techniques can be used effectively during our mission, as described below.

\section{Dawn's Observing Plans}

As discussed briefly above, Dawn collects multi-angle images in the Survey, HAMO-1 and HAMO-2 orbit sub-phases. All of Dawn's orbits are polar. In LAMO, images are collected only at nadir, but some deviation from nadir pointing is expected that will yield data with small off-nadir angles ( $<5$ degrees). To avoid the spacecraft entering the shadow of Vesta or Ceres, the orbit plane relative to the Sun must change as the spacecraft gets closer to the asteroid. For Vesta, the orbit beta angle, defined as the angle between the orbit plane and the Vesta-Sun plane, is $10-15^{\circ}$ in Survey, $30^{\circ}$ in HAMO- 1 , and $45^{\circ}$ in LAMO and 
Fig. 6 Image coverage in Survey for different phase angle bins. Up to the limit of the body's illumination conditions, all areas are covered with $>5$ independent views (images) with phase angle $>20^{\circ}$ with a few exceptions. Coverage below $20^{\circ}$ is still quite good

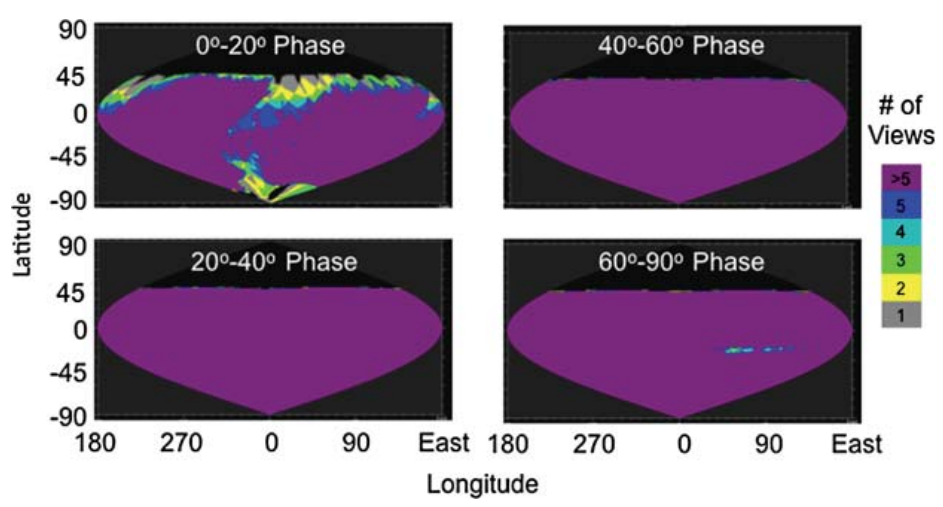

HAMO-2. The beta angle defines, approximately, the minimum phase angle, which occurs at the subsolar point. The beta angles were chosen to optimize the spectral reflectance data obtained by the visible and infrared (VIR) mapping spectrometer in Survey, and the FC mapping in HAMO-1. The LAMO orbit beta angle of $45^{\circ}$ is the minimum safe angle, and the constraints of the orbit transfer design dictated that HAMO-2 have the same beta angle as LAMO. More information on the science planning and operations can be found in Polanskey et al. (2011). The impact of this orbit design is that:

1. Illumination variation can be minimized, given the polar orbit, and is necessarily small given the limits on how large the off-nadir angle can be and stay on the body; and,

2. There is ample illumination variation across the combined set of orbit sub-phases.

The low beta angle in Survey yields large phase angle variation within the data set. Several sets of limb images are obtained in Survey, but otherwise there are no dedicated off-nadir images. SPC uses the Survey images to build a landmark network and derive a low-resolution shape model upon which the high-resolution shape models build as higher-resolution images are obtained. The stereo technique uses the Survey images to produce a low-resolution shape model. The phase angle coverage achieved in Survey is shown in Fig. 6. From these data, a shape model with 250-m horizontal spatial resolution and 10's of meters height accuracy can be derived using either technique.

The main topographic mapping data set is obtained in HAMO-1 and HAMO-2. Since the illumination variation is limited within a single sub-phase, the Stereo technique is expected to yield better results for the HAMO-1 data set alone. Based on the Virtual Vesta validation experiment described in Sect. 6, Stereo is expected to produce a DSM from HAMO-1 that achieves $10 \mathrm{~m}$ height resolution but that will be limited in extent in the northern hemisphere. HAMO-1 occurs during the height of the austral summer on Vesta, whose year is on the order of four Earth years, so the northern hemisphere is dark during the beginning of Dawn's stay at Vesta. Data useful and necessary for SPC to produce a DSM with comparable resolution will not be obtained until significantly different illumination conditions occur. For these reasons, amongst others, a second HAMO orbit is planned (HAMO-2) during departure, which will enable a high-resolution SPC model to be constructed. Stereo will also derive a separate model using HAMO-2 data to extend coverage to the north. Since SPC can exploit all the image data, of varying viewing conditions and resolution, it may surpass the height uncertainty of the Stereo model when all data (Survey, HAMO-1, LAMO, HAMO-2) are included. Once the Stereo and SPC DSMs are developed and validated, they will be assessed and merged with the gravity field to produce a Digital Topographic Model (DTM) that is referenced to the Vesta center-of-mass (Sect. 4.4).

In HAMO orbit, global mapping with the framing camera requires ten orbits over five days to complete; the image footprints relative to the ground track are shown in Fig. 7. 
Fig. 7 Illustration of the HAMO ground track for several orbits (green lines) showing framing camera footprints in pink. The subsolar point is the red triangle. Each orbit lays down an image swath with overlapping frames that abuts, or overlaps, the neighboring swath. In ten orbits, or roughly 5 days, Dawn has "mowed the lawn" over the whole body and starts over

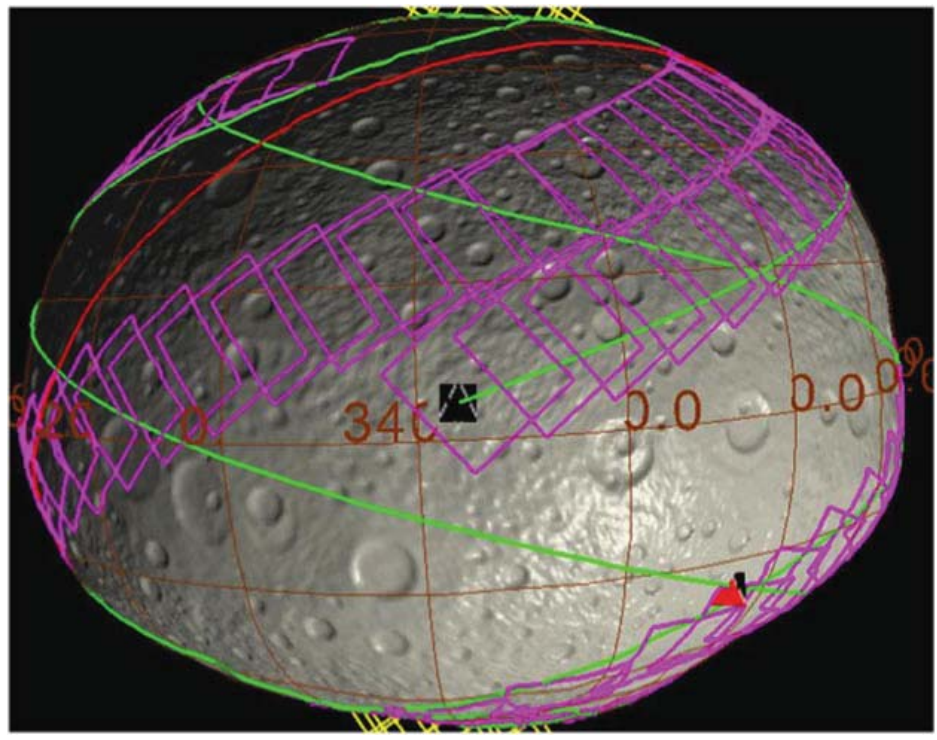

Fig. 8 Illustration of the off-nadir viewing geometry in HAMO-1 (left) and HAMO-2 (right) as referenced to spacecraft vector coordinates for the six global mapping cycles of HAMO-1 (C1-C6) and four cycles of HAMO-2 (C1-C4). For Dawn's polar orbit, constant illumination is in the "Ahead" direction, while maximum illumination variation is achieved in the "Cross" direction. The angles in the Cross direction are maximized within the constraints of keeping the frames on the body and achieving proper incidence and emission angles. The approximate direction of the Sun is shown for equatorial latitudes
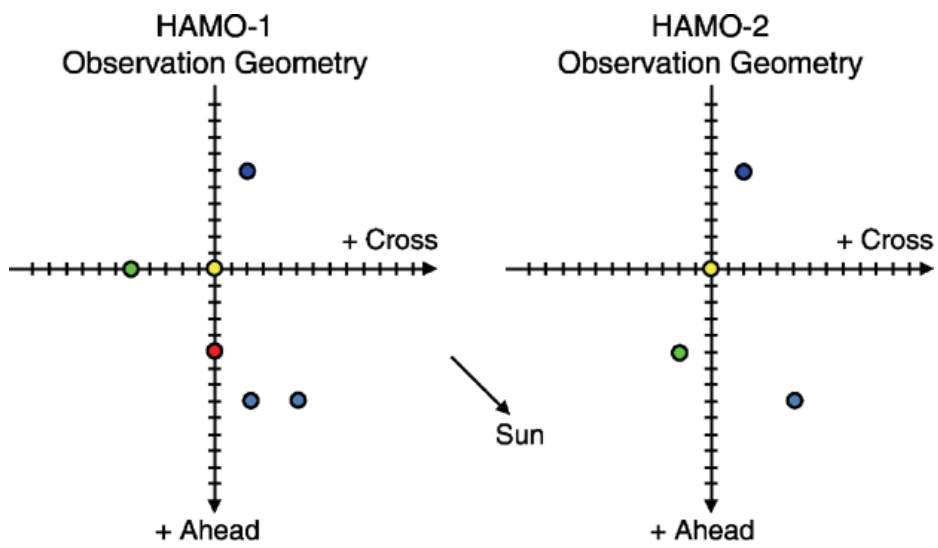

o C1 Nadir

- C2 Ahead+8/Cross+5 (N/E) and Ahead+8/Cross+2 (S)

- C3 Ahead-6/Cross+2

- C4 Ahead+5

o C5 Cross-5

Note that the rapid rotation of Vesta (period 5.34 hours) yields a spiral ground track for Dawn's polar orbit. The plans for HAMO-1 include four off-nadir global mapping cycles and two nadir mappings; each off-nadir global mapping is done at a fixed attitude. The Dawn spacecraft has an attitude control mode in which a fixed off-nadir attitude can be specified with respect to the spacecraft motion vector, by specifying the number of degrees off-nadir the pointing vector is in the direction of motion (ahead) and the cross-track direction (cross). The off-nadir angles were chosen such that three of the four angles would be useful for each technique (and one angle would be essentially useless for each technique). In HAMO-2, we optimized the off-nadir angles to favor Stereo, as that technique cannot exploit any data resulting from the combination of the two sub-phases. The viewing geometry is shown graphically in "Ahead-Cross-Nadir" coordinates in Fig. 8. Coverage plots for Stereo, from combined HAMO-1 and 2 coverage, are shown in Fig. 9, which also illustrates the fact that most HAMO data that satisfy the Stereo illumination variation conditions have a very narrow range of illumination, $<5^{\circ}$ (bottom panel), which is a natural consequence of 


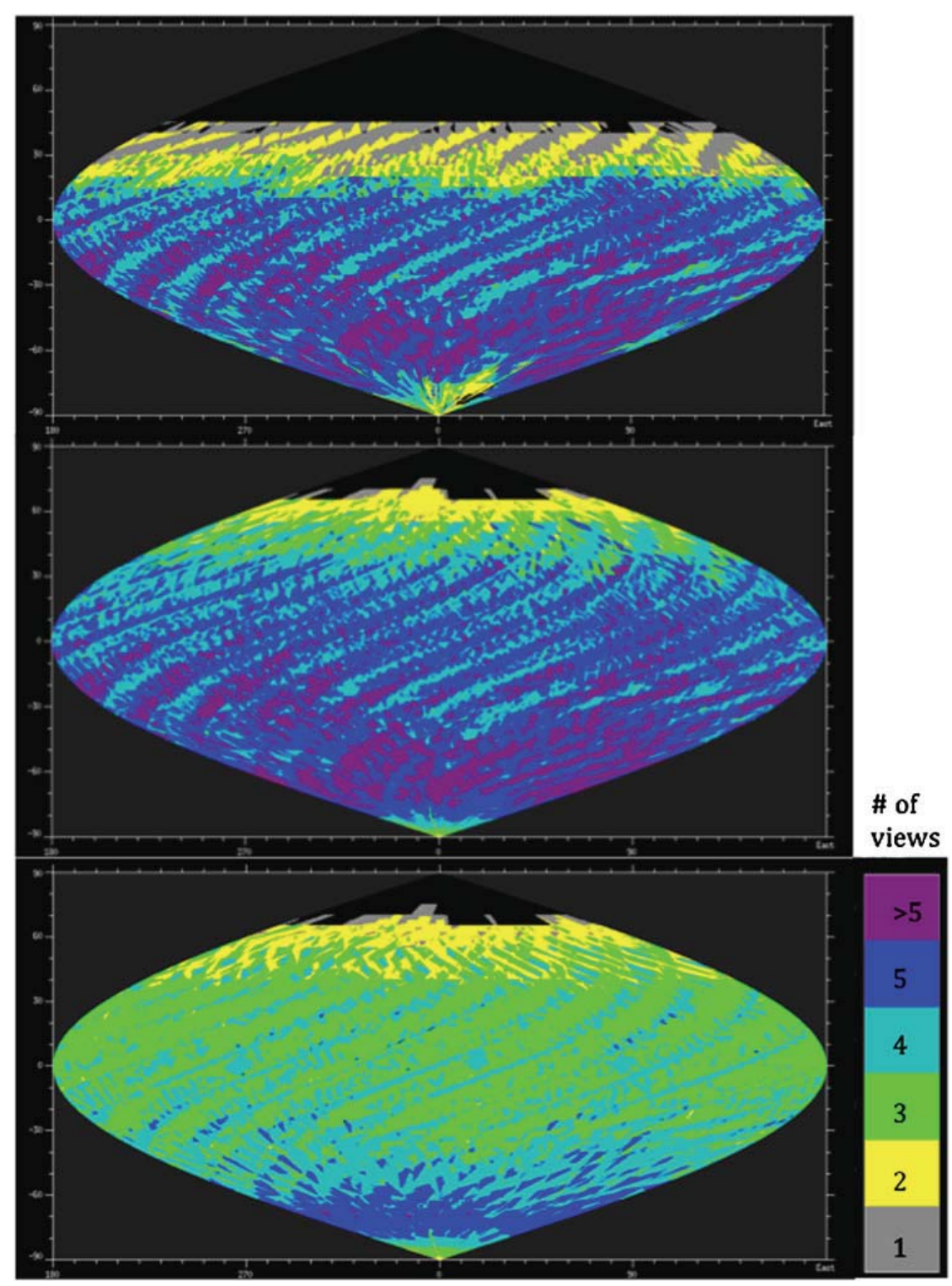

Fig. 9 Coverage plots for optimal stereo conditions (top) and the same but with incidence angle range $0^{\circ}$ to $80^{\circ}$ (center). The bottom plot shows coverage for incidence angle range $0^{\circ}$ to $80^{\circ}$ and illumination variation of $<5^{\circ}$, indicating the high quality of the image pairs. The colors indicate the number of independent datums in each $0.25^{\circ}$ bin that collectively meet the criteria outlined in Table 1 . The area covered at a given number of views is reported in Table 2

the polar sun-synchronous orbit. In Fig. 10, the coverage for SPC is shown, for both HAMO1 alone, and HAMO- 1 and 2 combined, illustrating that coverage is poor when considering only HAMO-1. The addition of HAMO-2 yields many useable data, because image pairs from different sub-phases contribute to the solution.

At least three views of the surface are required to derive a robust height estimate, and additional views generally improve the accuracy. These coverage analyses show that sufficient data will be collected to model the topography over $>80 \%$ of the surface of Vesta. Although relaxation of the optimal incidence range is required to meet the coverage requirement for stereo, the quality of the data as evidenced by the narrow range of illumination variation (Fig. 9, bottom panel) indicates that the height requirement can be met. Data that meet the useable criteria in Table 1 will also be used to extend coverage to the northern region, al- 


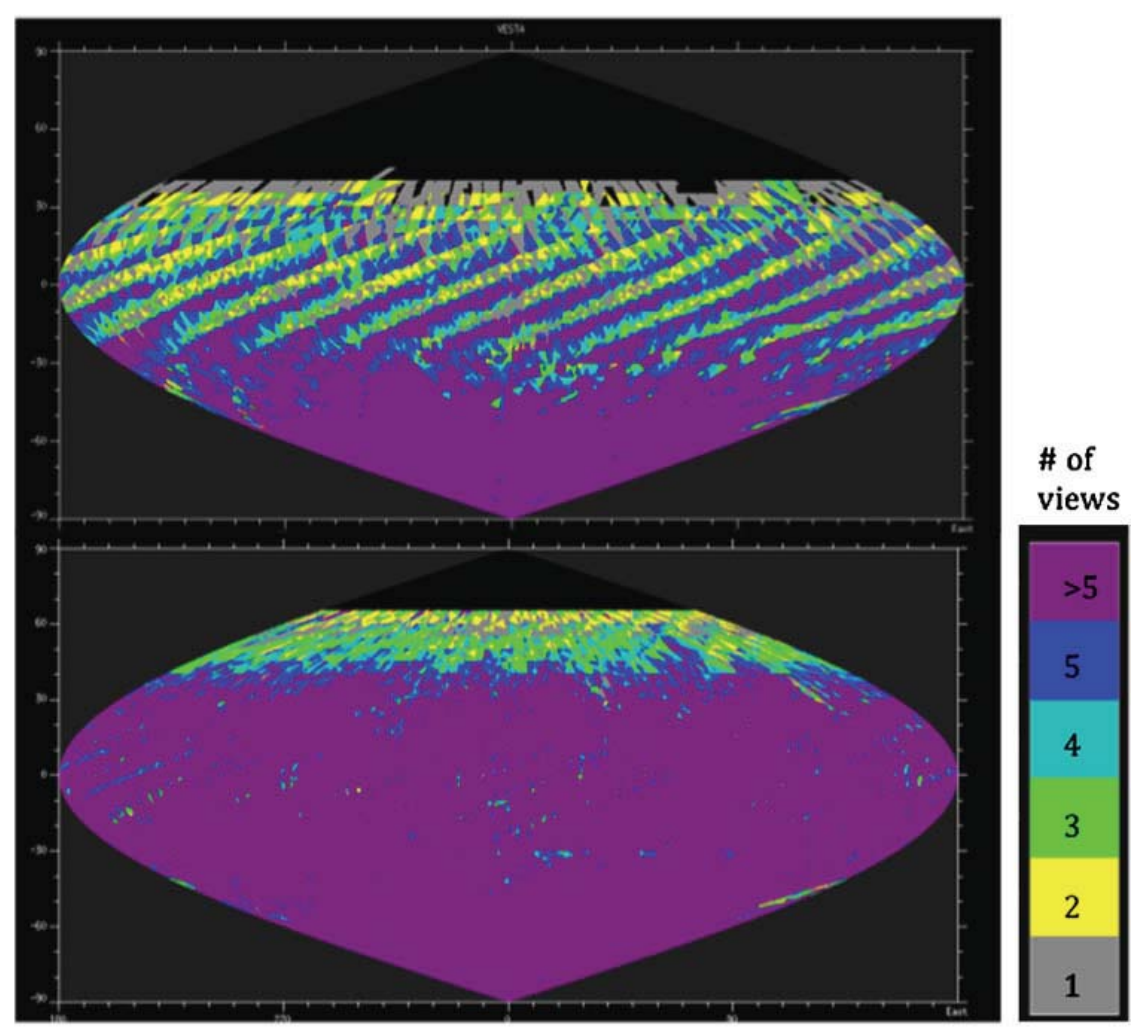

Fig. 10 Coverage plot for optimal SPC conditions for HAMO-1 data alone (top) and the combined HAMO-1 and HAMO-2 data (bottom). See Fig. 9 caption for key and Table 2 for areal coverage. Coverage is sparse north of $30^{\circ} \mathrm{S}$ for HAMO-1 data. Excellent coverage is obtained to $\sim 60^{\circ} \mathrm{N}$ in the combined HAMO-1 and HAMO-2 data set

though the height accuracy may be reduced. Validation that the data quality is sufficient to meet the 10-m height accuracy requirement for the primary data set is discussed in Sect. 6, which reports the results of a simulation validation experiment.

\section{Virtual Vesta}

The Dawn optical navigation team provided a virtual dataset comprising an early version of the Survey and HAMO data planned to be acquired at Vesta. The virtual dataset consisted of 507 Survey images covering Vesta from the south pole to $53^{\circ} \mathrm{N}$ at a mean resolution of 250 meters/pixel, and 2005 HAMO images from the south pole to $58^{\circ} \mathrm{N}$ at a mean resolution of 60 meters/pixel. The Virtual Vesta data set deviates from the actual mission plan in two ways: The HAMO orbit altitude for Virtual Vesta is slightly lower than the planned HAMO altitude, yielding about $15 \%$ better image resolution, and the number of HAMO images used is lower than the actual plan: 2005 versus 2500; the Virtual Vesta plan had only three offnadir mappings in HAMO-1, whereas the current plan has four. In addition, an important difference and one that resulted in part from the results of Virtual Vesta, is that HAMO-2 was not included in the exercise, as it was not part of the baseline plan when the simulated data sets were created.

The simulated Vesta surface is based on the HST-derived global shape by Thomas et al. (1997), with the addition of craters based on a power-law distribution with exponent -2.2 , and a diameter range of $0.4-100 \mathrm{~km}$; large incomplete craters are similar to rilles and cuts 
Fig. 11 A rendering of the Virtual Vesta model

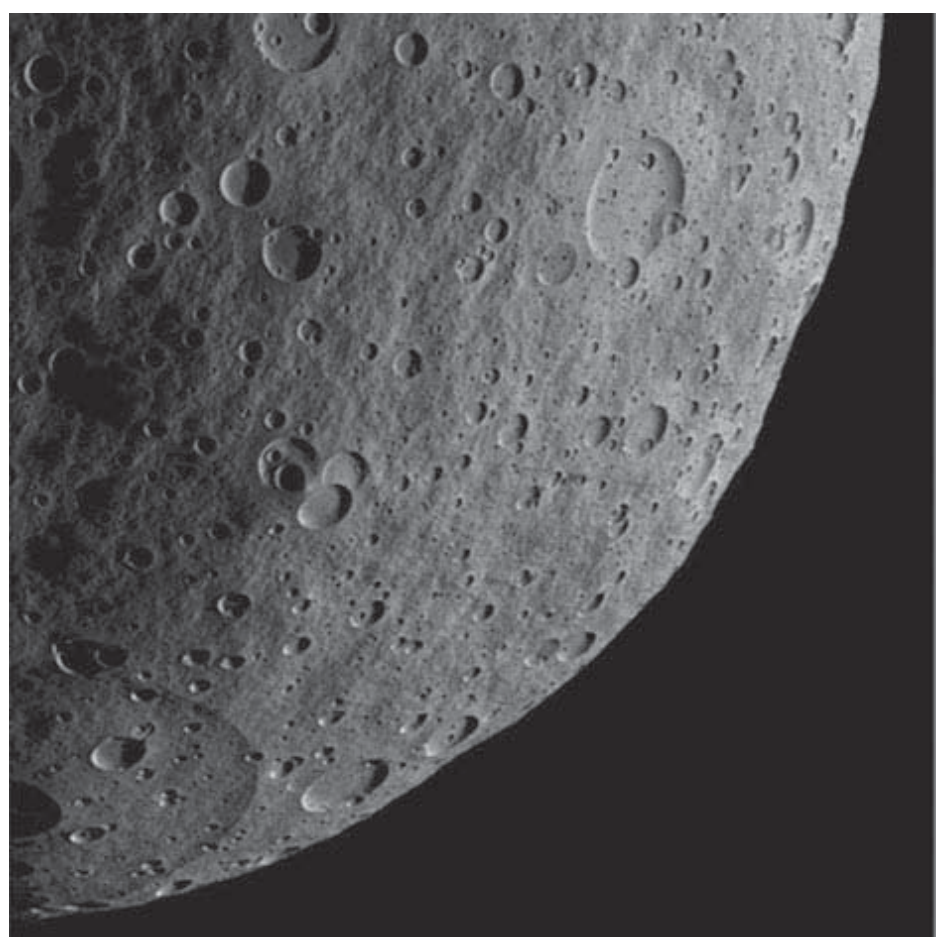

across the surface. Surface perturbations at wavelengths from a few hundred meters to tens of $\mathrm{km}$ were also present. The resolution of the Virtual Vesta model is $10 \mathrm{~m} / \mathrm{pixel}$ with a height accuracy of $\sim 1 \mathrm{~m}, 1$-sigma. The simulated trajectory and camera orientation data contain errors whose magnitude is consistent with what is expected at a post-processing stage: random orientation error of one FC pixel 1-sigma, per image, and correlated position errors that range from a few $\mathrm{km}$ in Survey to a few hundred meters in HAMO. Both orbits consist of highly variable illumination geometry (Sun azimuth: $0-360^{\circ}$, incidence: $0-90^{\circ}$ ) for the illuminated part of Vesta (i.e. the southern hemisphere up to mid northern latitudes) at the time period of the respective orbits. In Survey, illumination variation is large for available image pairs due to the large camera footprint, while for HAMO illumination variation within image pairs in small, because it is dominated by the polar orbit. Slowly varying albedo fluctuations were also introduced to further simulate the actual data set. This synthetic dataset was used for making software adaptations, to test both the Stereo and SPC processing techniques, and to validate that the observing plan would be sufficient to meet the height accuracy requirement of $10 \mathrm{~m} / \mathrm{pixel}$ at $100 \mathrm{~m} / \mathrm{pixel}$ horizontal resolution. An illustration of a portion of the synthetic Virtual Vesta surface is shown in Fig. 11.

The large illumination variation within the Survey images provides the foundation of the SPC model. In contrast, Survey is not well suited for stereophotogrammetric mapping. Nevertheless, images from this mission phase provide sufficient stereo overlap, so that stereo processing under useable conditions can be performed for the entirely imaged area. Simulated HAMO-1 data, with their higher resolution and multi-angle viewing geometry provide the main data set for the DSMs. DSMs for Survey and HAMO data were created by using SPC and Stereo, and these model results were then compared to the truth model to determine the height accuracy expected for this observing plan and conditions.

For the Stereo models, the photogrammetric processing line that was previously described, from block adjustment of orientation data up to the interpolation of a DSM of the covered surface, was applied successfully. For all processing steps the full observation and illumination restrictions have been taken into account. Figure 12 shows a typical pair of 

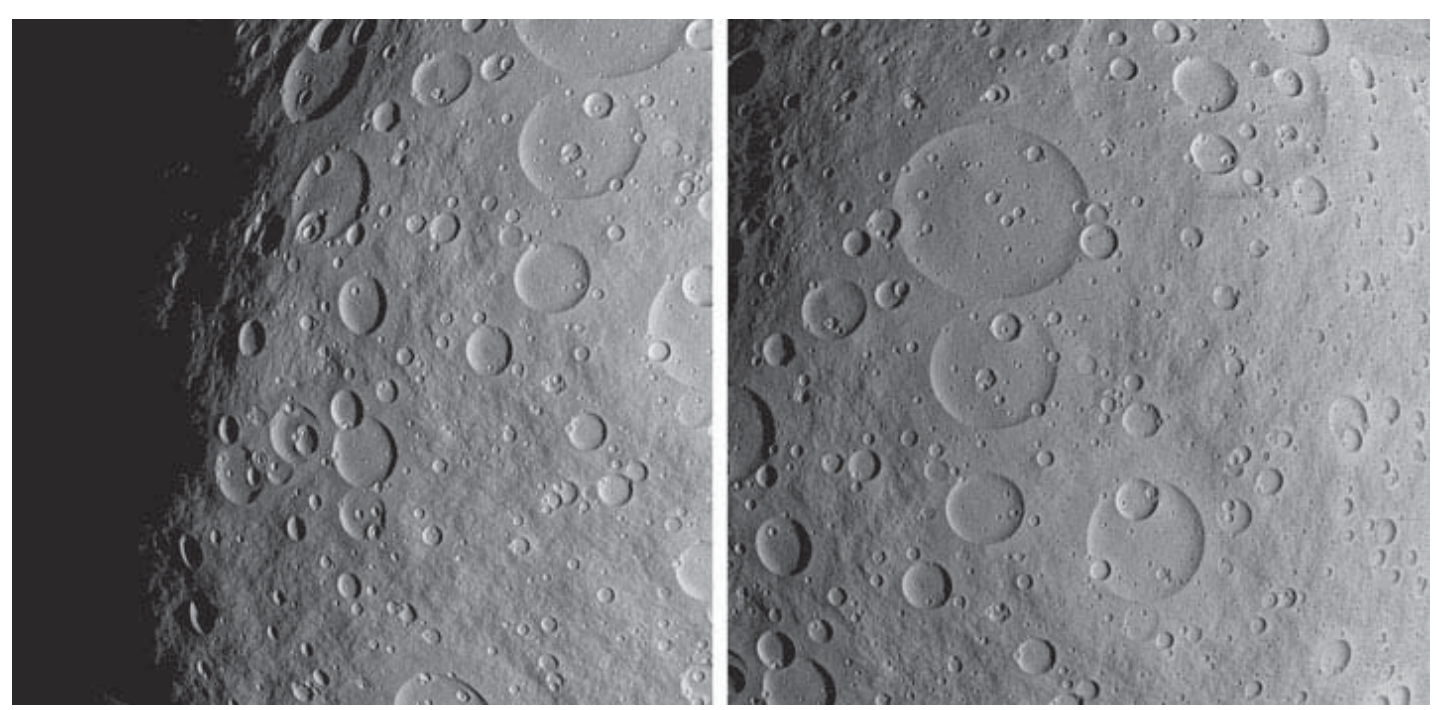

Fig. 12 Overlapping pair of Survey images from Virtual Vesta dataset
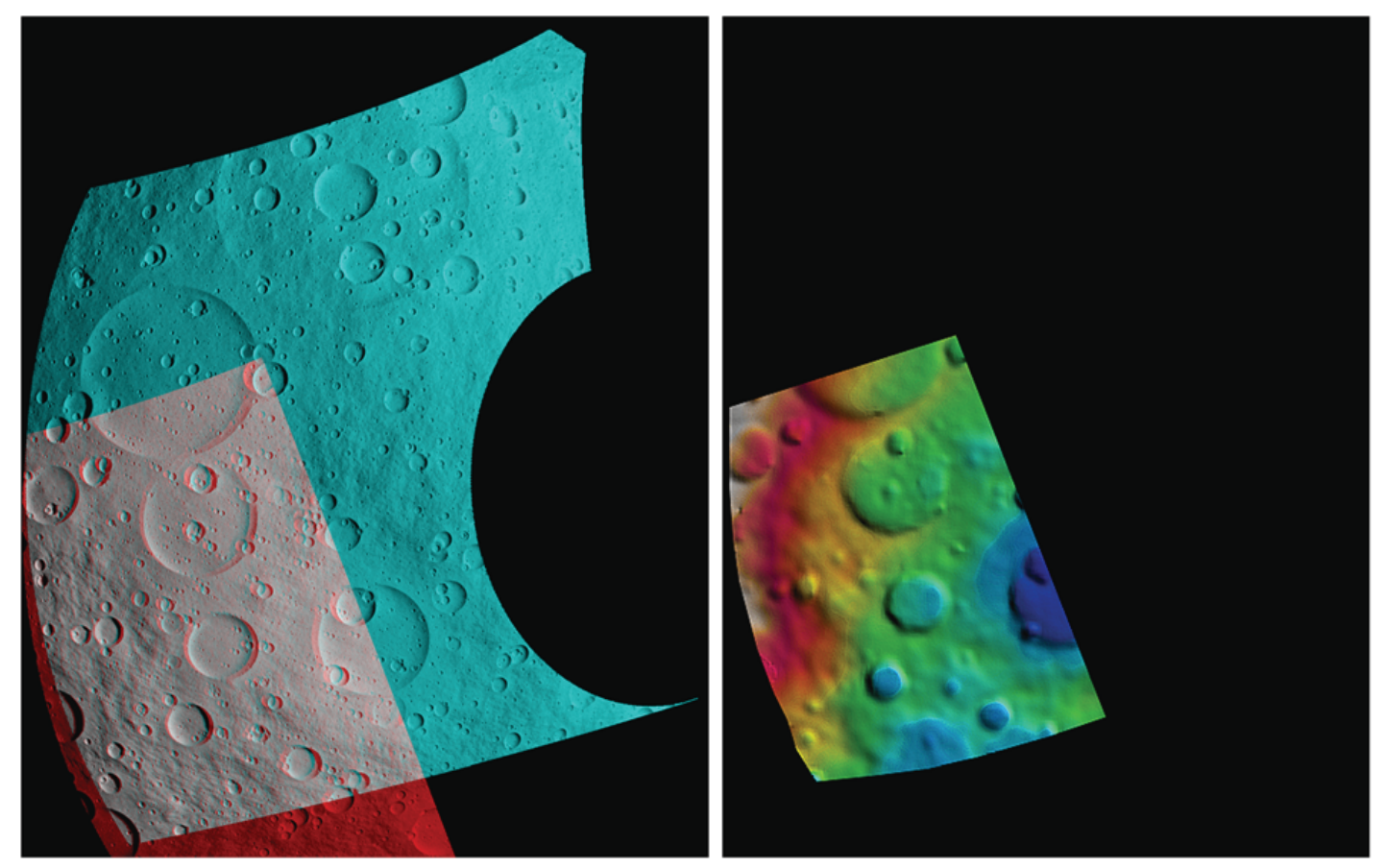

Fig. 13 Left: Pre-rectified pair of Survey images from Virtual Vesta dataset. Right: DSM of stereo overlap. Height range above 3-axial ellipsoid: $-9 \mathrm{~km}$ (blue) to $7.3 \mathrm{~km}$ (pink/gray)

stereo images (raw data). Figure 13 (left) shows the pre-rectified images (initially rectified to a constant height). Following the illumination restrictions, areas with emission angle $>75^{\circ}$ (left side) are excluded, as well as areas with incidence angle $<20^{\circ}$ (right circle). Figure 13 also provides a DSM from this pair's stereo overlap (right). From the overall virtual dataset more than 2.7 billion object points have been derived and a final DSM has been interpolated (Fig. 14). For Survey, the mean forward ray intersection accuracy of all object points is $\pm 39 \mathrm{~m}$, i.e. $16 \%$ of the mean FC ground resolution, and for the HAMO data set is $\pm 6 \mathrm{~m}$, i.e. $10 \%$ of the mean ground resolution. 
Fig. 14 Stereo DSM from Survey and HAMO simulated Virtual Vesta data. The model extends from $90^{\circ} \mathrm{S}$ to $47^{\circ} \mathrm{N}(87 \%$ of surface) at $125 \mathrm{~m} /$ pixel resolution. The model has 2.7 billion points and a mean point accuracy of $6.0 \mathrm{~m}(3 \mathrm{D}$, 1-sigma). Heights range from $-56.3 \mathrm{~km}$ (blue) to $23.4 \mathrm{~km}$ (white) over a sphere with a $270 \mathrm{~km}$ radius
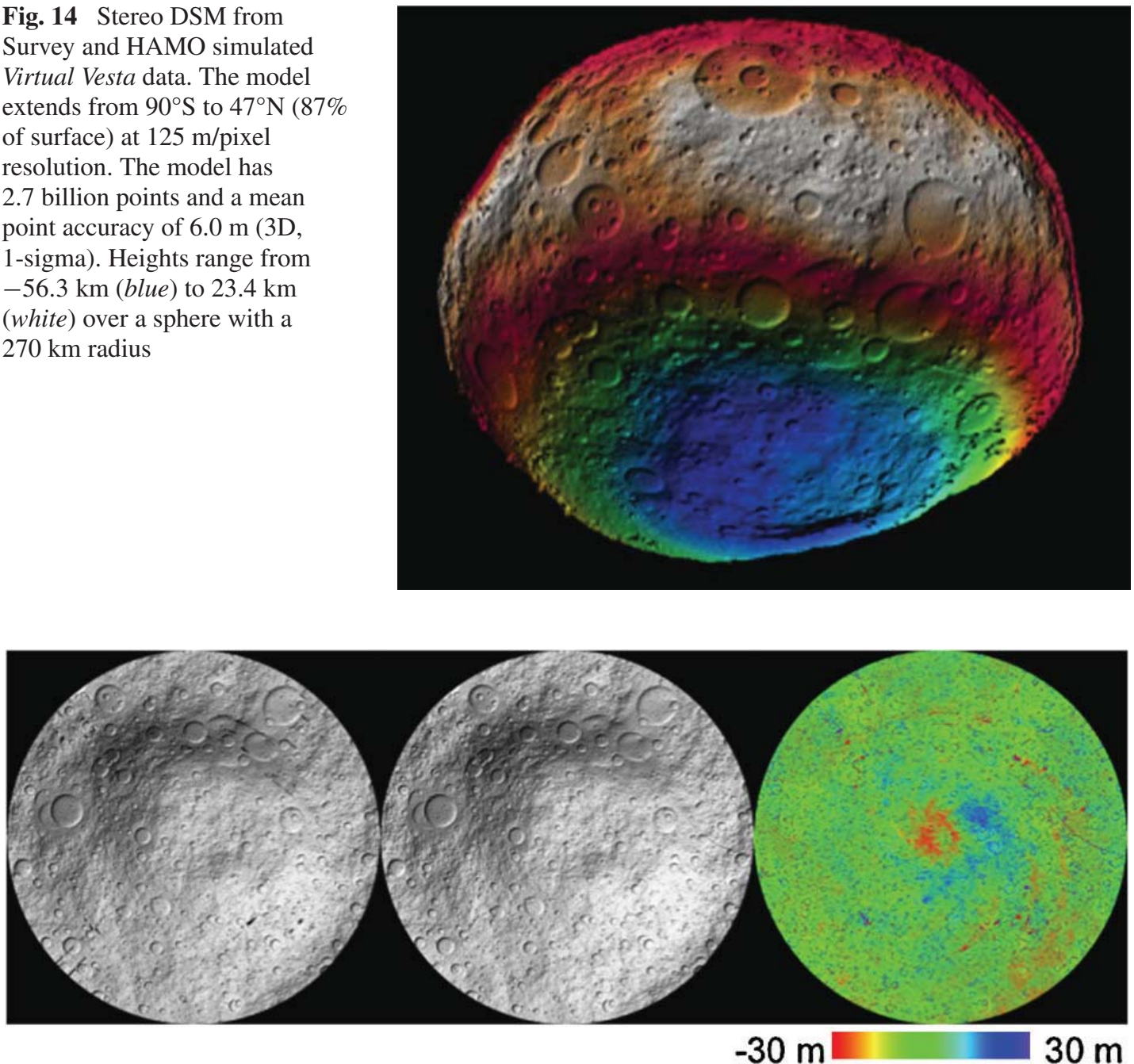

Fig. 15 Left: Virtual Vesta (truth) model. Middle: Shaded relief 125-m raster DSM in Stereographic projection (south pole at center, latitudes up to $0^{\circ} \mathrm{N}$ ), derived by stereophotogrammetric reconstruction from synthetic FC images of Dawn's Survey and HAMO-1 orbits. Right: Residuals after subtracting the truth model. Heights range from-30 $\mathrm{m}$ (red) to $30 \mathrm{~m}$ (blue)

\subsection{Comparison with Truth Model}

Comparison of the derived HAMO DSM to the truth model (Fig. 15) yields an agreement within $\pm 30 \mathrm{~m}$ over $80 \%$ of the surface between $0^{\circ}$ and $90^{\circ} \mathrm{S}$, an area with robust image coverage. Histograms of the statistical comparisons of both the Stereo and SPC HAMO models to the truth model, calculated over the full range of the illuminated surface (to $47^{\circ} \mathrm{N}$ ), are shown in Fig. 16. The SPC errors (top panel) are larger than those for Stereo (bottom). The percentage of errors for the Stereo model that are $<20 \mathrm{~m},<30 \mathrm{~m}$ and $<40 \mathrm{~m}$ are $41.8 \%, 58.7 \%$ and $71.6 \%$ respectively, while for SPC, $27 \%$ of errors are $<20 \mathrm{~m}, 40 \%$ are $<30 \mathrm{~m}, 52 \%$ are $<40 \mathrm{~m}$, and $62 \%$ are $<50 \mathrm{~m}$. The SPC model produced using the simulated Survey and HAMO data is not as accurate because the Virtual Vesta data set lacks substantial illumination variation within the HAMO-1 orbit, which drives SPC accuracy at the 100-m horizontal resolution. To illustrate this point, Fig. 17 shows maps of the imaging coverage with optimal conditions as specified in Table 1 for SPC and Stereo. The optimal conditions for SPC are met only in the high southern latitudes, while coverage for Stereo is adequate 
Fig. 16 Cumulative distributions of absolute height errors calculated as the difference between the height of the derived Stereo and SPC models and the Virtual Vesta truth model. The errors are calculated for each model point using bi-linear interpolation of the four nearest neighbor Virtual Vesta heights

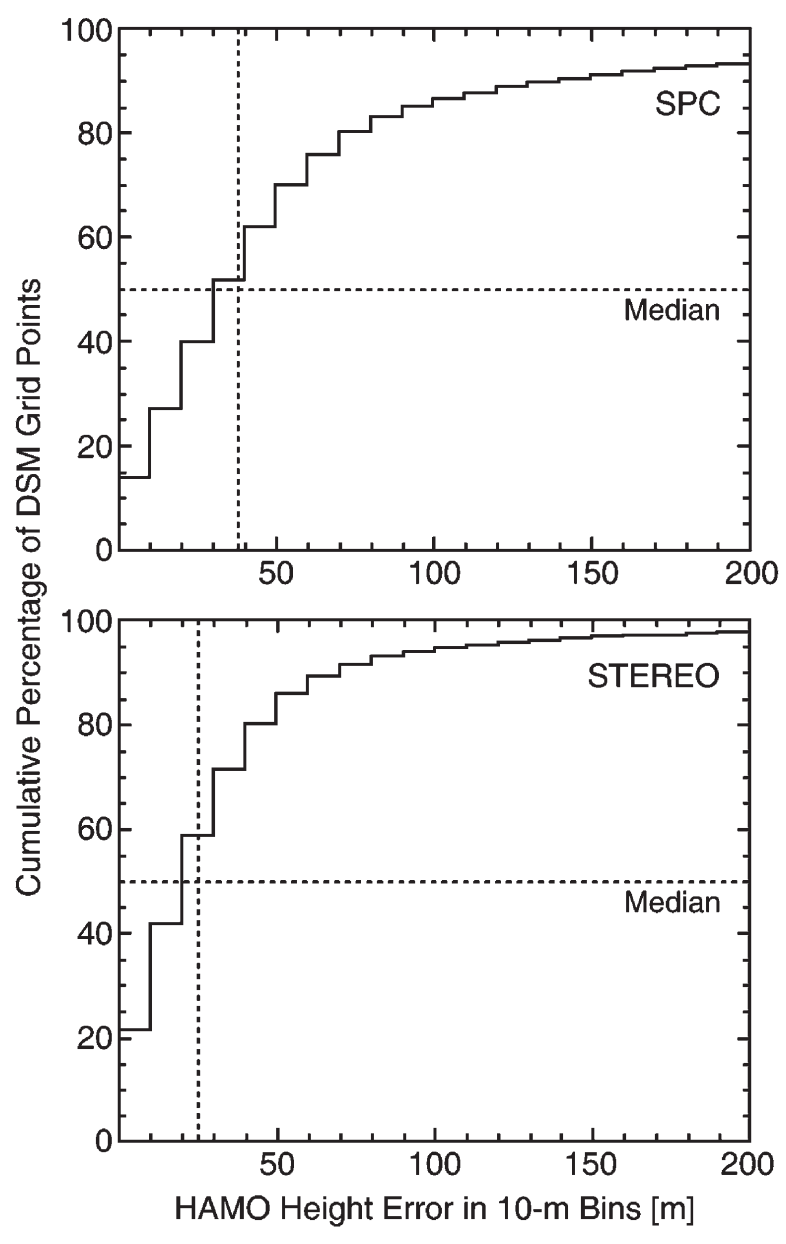

over most of the surface to $20^{\circ} \mathrm{N}$, explaining the large deviations from the truth model for the SPC.

\subsection{Expected Accuracy}

Dawn's imaging plans for off-nadir data were modified as a result of the Virtual Vesta exercise. However, the Virtual Vesta experiment was not repeated with the current plan that has increased imaging in HAMO-1 with refined off-nadir angles, and a HAMO-2 orbit. The results described above for comparison of the DSMs derived from the simulated Virtual Vesta data and the truth model were examined to infer the accuracy expected for the actual imaging plans. For Stereo, the percent coverage of the surface with three views meeting the optimal conditions (Fig. 17, bottom) was 55.7\%, and the height accuracy relative to the truth model was $58.7 \%$ at $<30 \mathrm{~m}$, yielding a roughly linear correlation between the coverage and height accuracy. If we compare to the expected coverage of the surface for the current imaging plans in HAMO-1 and HAMO-2, with incidence up to $80^{\circ}$ (Table 2), we predict that the DSM should be accurate to $<30$ m over roughly $87 \%$ of the surface, while the relative accuracy should be comparable to the \pm 6 m mean forward ray intersection accuracy. Improved accuracy is expected to result from the higher number of views per bin in the current plan relative to Virtual Vesta. Actual conditions will dictate the final accuracy. The surface coverage might also improve with inclusion of data outside the optimal range.

The illumination variation between HAMO-1 and HAMO-2 results in a data set that satisfies SPC optimal conditions over nearly the entire lit surface (Fig. 10, bottom). Analysis 
Table 2 The surface coverage obtained for the combination of HAMO-1 and HAMO-2, as a function of the number of data (views) per 0.25 degree bin, under various conditions for the Stereo technique, and optimal conditions for the SPC technique. The color code in the column headings corresponds to the color scale in Figs. 9, 10, and 17

\begin{tabular}{|c|c|c|c|c|c|c|}
\hline \multirow{2}{*}{ Technique—conditions } & \multicolumn{6}{|c|}{ Area $(\%)$ covered with specified \# of views } \\
\hline & $>5$ & 5 & 4 & 3 & 2 & 1 \\
\hline Stereo-optimal & 13.8 & 42.4 & 59.9 & 63.7 & 71.5 & 79.2 \\
\hline Stereo- $-0-80^{\circ}$ incidence & 16.9 & 53.5 & 80.6 & 87.1 & 93.1 & 94.8 \\
\hline Stereo- $-0-80^{\circ}$ inc.; $<5$ deg. IV & 0.1 & 9.9 & 43.4 & 83.4 & 92.6 & 94.0 \\
\hline SPC—optimal & 73.7 & 77.9 & 82.9 & 87.4 & 89.6 & 92.5 \\
\hline
\end{tabular}

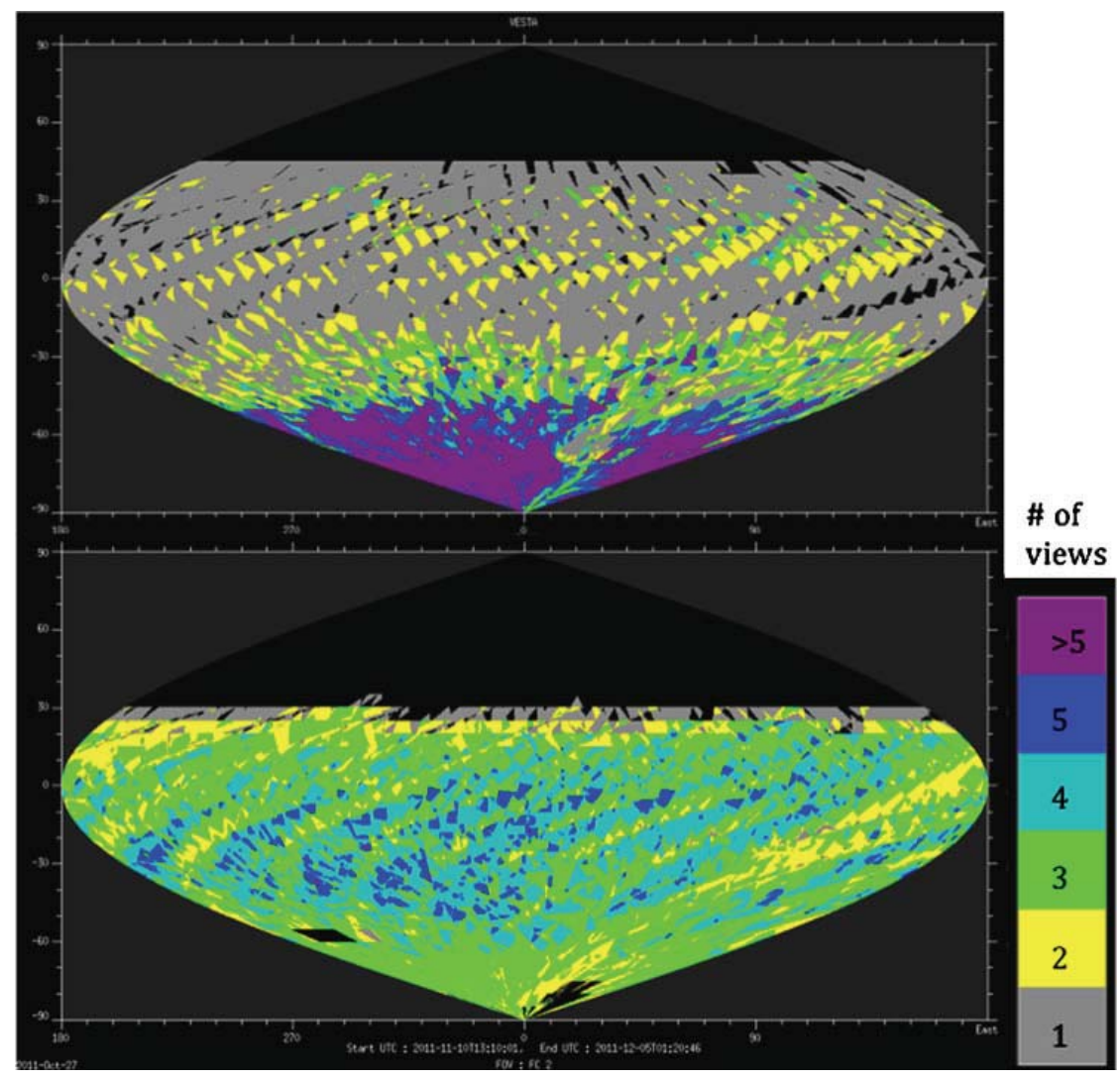

Fig. 17 Virtual Vesta coverage for SPC (top) and Stereo (bottom)

of this robust data set is expected to meet a height accuracy of $<10 \%$ of the image resolution, or $\sim 6 \mathrm{~m}$, by analogy with previous models (Gaskell et al. 2008).

The Virtual Vesta exercise was useful to test the SPC and Stereo techniques with simulated data, and to get a quantitative measure of the expected accuracy of the derived DSMs given Dawn's observing plans. The results were used to validate the plans in terms of the number of off-nadir data required, and their attitudes, by scaling the Virtual Vesta accuracy results for Stereo using coverage as a metric. It also proved the need for a second HAMO campaign late in the Vesta encounter to allow mapping in the far northern latitudes, and at different illumination conditions, to extend coverage and realize the full accuracy of the SPC method. As a result, the combination of the Stereo and SPC models in expected to produce 
a robust result over the illuminated surface, ensuring that our mapping requirement of $10 \mathrm{~m}$ height accuracy at $<100 \mathrm{~m} /$ pixel resolution over $>80 \%$ of Vesta is met.

\section{Expected Results}

The high-resolution digital shape models derived using various techniques and merged into a geodetically controlled topographic model will enable investigation of the many geologic landforms expected on Vesta. Volcanic and tectonic features will be able to be resolved at the scale of dikes and individual lava flows. Slumps and landslides can be identified and tied to source regions upslope. Craters of several hundred meters diameter will be well resolved topographically and their degradation states revealed. The high-resolution slope data will enable brightness variations to be confidently identified and related to spectral variations in terms of composition, grain sizes, or other factors.

Dawn's high-resolution topographic models, together with the gravity data, will reveal the tectonic, volcanic and impact history of Vesta, and enable co-registration of data sets to determine Vesta's geologic history. While the surface of Ceres is not expected to be as complex as Vesta's, the topographic mapping will likely reveal much about processes of surface modification as well as the internal structure and evolution of this dwarf planet.

Acknowledgements The authors are grateful for support of the Virtual Vesta analysis by the Dawn project team, and for the comments of two anonymous reviewers, which improved the paper. A portion of this work was performed at the Jet Propulsion Laboratory, California Institute of Technology, under contract with NASA.

\section{References}

R.P. Binzel, S. Xu, Chips off of Asteroid 4 Vesta: Evidence for the parent body of basaltic achondrite meteorites. Science 260, 186-191 (1993)

C. Capanna, L. Jorda, P.L. Lamy, G. Gesquiere, 3D reconstruction of solar system bodies using photoclinometry by deformation, in Proceedings of the International Conference on Computer Graphics, Visualization, Computer Vision and Image Processing, Rome (2011)

B. Carry, C. Dumas, M. Fulchignoni, W.J. Merline, J. Bertheir, D. Hestroffer, T. Fusco, P. Tamblyn, Nearinfrared mapping and physical properties of the dwarf-planet Ceres. Astron. Astrophys. 478, 235-244 (2008)

J.C. Castillo-Rogez, T.B. McCord, Ceres' evolution and present state constrained by shape data. Icarus 205, 443-459 (2010). doi:10.1016/j.icarus.2009.04.008

J.F. Cavanaugh, J.C. Smith, X. Sun, A.E. Bartels, L. Ramos-Izquierdo, D.J. Krebs, J.F. McGarry, R. Trunzo, A. Novo-Gradac, J.L. Britt et al., The Mercury laser altimeter instrument for the MESSENGER mission. Space Sci. Rev. 131, 451-479 (2007). doi:10.1007/s11214-007-9273-4

J. Drummond, J. Christou, Triaxial ellipsoid dimensions and rotational poles of seven asteroids from Lick Observatory adaptive optics images, and of Ceres. Icarus 97, 480-496 (2008)

M.J. Gaffey, Surface lithologic heterogeneity of Asteroid 4 Vesta. Icarus 127, 130-157 (1997)

R.W. Gaskell, O.S. Barnouin-Jha, D.J. Scheeres, A.S. Konopliv, T. Mukai, S. Abe, J. Saito, M. Ishiguro, T. Kubota, T. Hashimoto, J. Kawaguchi, M. Yoshikawa, K. Shirakawa, T. Kominato, N. Hirata, H. Demura, Characterizing and navigating small bodies with imaging data. Meteoritics and Planetary. Science 43, 1049-1062 (2008)

R.W. Gaskell, Optical navigation near small bodies. AAS paper 11-220, AAS/AIAA Space Flight Mechanics Meeting, New Orleans, LA (2011)

B. Giese, T. Denk, G. Neukum, T. Roatsch, P. Helfenstein, P. Thomas, E. Turtle, A. McEwen, C. Porco, The topography of Iapetus' leading side. Icarus 193, 359-371 (2007)

R. Greeley, G. Batson, Planetary Mapping (Cambridge University Press, Cambridge, 1990)

K. Gwinner, F. Scholten, M. Spiegel, R. Schmidt, B. Giese, J. Oberst, C. Heipke, R. Jaumann, G. Neukum, Derivation and validation of high-resolution digital topographic models from Mars express HRSC data. Photogramm. Eng. Remote Sens. 75, 1127-1142 (2009a) 
K. Gwinner, F. Scholten, F. Preusker, S. Elgner, T. Roatsch, M. Spiegel, R. Schmidt, J. Oberst, R. Jaumann, C. Heipke, Topography of Mars from global mapping by 17 HRSC high-resolution digital topographic models and orthoimages: characteristics and performance. Earth Planet. Sci. Lett. (2009b). doi:10.1016/j.eps1.2009.11.007

S.E. Hawkins III, J.D. Boldt, E.H. Darlington, M.P. Grey, C.J. Kardian Jr., S.L. Murchie, K. Peacock, E.D. Schaefer, B.D. Williams, Overview of the MESSENGER Mercury dual imaging system, in 33rd Lunar and Planetary Science Conference, vol. 8041 (2001)

R. Jaumann, G. Neukum, T. Behnke, T.C. Duxbury, J. Flohrer, S. v. Gasselt, B. Giese, K. Gwinner, E. Hauber, H. Hoffmann, U. Köhler, K.-D. Matz, T.B. McCord, V. Mertens, J. Oberst, R. Pischel, D. Reiss, T. Roatsch, P. Saiger, F. Scholten, G. Schwarz, K. Stephan, M. Wählisch, The High Resolution Stereo Camera (HRSC) experiment on Mars express: instrument aspects and experiment conduct from interplanetary cruise through the nominal mission. Planet. Space Sci. 55, 928-952 (2007)

A.S. Konopliv, S.W. Asmar, B.G. Bills, N. Mastrodemos, R.S. Park, C.A. Raymond, D.E. Smith, M.T. Zuber, The Dawn gravity investigation at Vesta and Ceres. Space Sci. Rev. (2011). doi:10.1007/s11214-011-9794-8

J.-Y. Li, L.A. McFadden, J.Wm. Parker, E.F. Young, P.C. Thomas, C.T. Russell, M.V. Sykes, S.A. Stern, Photometric analysis of 1 Ceres and surface mapping from HST observations. Icarus 182, 143-160 (2006)

J.-Y. Li, L.A. McFadden, P.C. Thomas, M.J. Mutchler, J.W. Parker, E.F. Young, C.T. Russell, M.V. Sykes, B.E. Schmidt, Photometric mapping of Asteroid (4) Vesta's southern hemisphere with Hubble Space Telescope. Icarus 208, 238-251 (2010)

T.B. McCord, J.B. Adams, T.V. Johnson, Asteroid Vesta: spectral reflectivity and compositional implications. Science 168, 1445-1447 (1970)

T.B. McCord, C. Sotin, Ceres: Evolution and current state. J. Geophys. Res. 110, E05009 (2005)

G.A. Neumann, D.D. Rowlands, F.G. Lemoine, D.E. Smith, M.T. Zuber, Crossover analysis of Mars Orbiter Laser Altimeter data. J. Geophys. Res. 106, 23,753-23,768 (2001). doi:10.1029/2000JE001381

J. Oberst, F. Scholten, K.-D. Matz, T. Roatsch, M. Wählisch, I. Haase, P. Gläser, K.K. Gwinner, M.S. Robinson (The LROC Team), Apollo17 landing site topography from LROC NAC stereo data-first analysis and results, in 41st Lunar and Planetary Science, vol. 2051 (2009)

D.E. Pavlis et al., GEODYN operations manuals. Raytheon ITTS Contractor Report, Lanham, MD (2001)

C.M. Pieters, L.A. McFadden, T. Prettyman, M.C. De Sanctis, T.B. McCord, T. Hiroi, R. Klima, J.-Y. Li, R. Jaumann, Surface composition of Vesta: issues and integrated approach. Space Sci. Rev. (2011). doi:10.1007/s11214-011-9809-5

C.A. Polanskey, S.P. Joy, C.A. Raymond, Dawn science planning, operations and archiving. Space Sci. Rev. (2011). doi:10.1007/s11214-011-9855-Z

F. Preusker et al., Stereo topographic models of Mercury after three MESSENGER flybys. Planet. Space Sci. (2011). doi:10.1016/j.pss.2011.07.005

V. Reddy, M.J. Gaffey, M.S. Kelley, A. Nathues, J.-Y. Li, R. Yarbrough, Compositional heterogeneity of Asteroid 4 Vesta's southern hemisphere: implications for the Dawn mission. Icarus 210, 693-706 (2010)

T. Roatsch, M. Wählisch, B. Giese, A. Hoffmeister, K.-D. Matz, F. Scholten, A. Kuhn, R. Wagner, G. Neukum, P. Helfenstein, C. Porco, High-resolution Enceladus atlas derived from Cassini-ISS images. Planet. Space Sci. 56, 109-116 (2008)

D.D. Rowlands et al., GEODYN II system description. Hughes-STX Contractor Report, Greenbelt, MD (1993)

C.T. Russell, C.A. Raymond, The Dawn discovery mission to Vesta and Ceres. Space Sci. Rev. (2011). doi:10.1007/s11214-011-9836-2

F. Scholten, K. Gwinner, Operational parallel processing in digital photogrammetry-strategy and results using different multi-line cameras, in Proceedings ISPRS XXth Congress, vol. 35(B) (2004), pp. 408413

F. Scholten, K. Gwinner, T. Roatsch, K.-D. Matz, M. Wählisch, B. Giese, J. Oberst, R. Jaumann, G. Neukum, HRSC co-investigator team Mars express HRSC data processing-methods and operational aspects. Photogramm. Eng. Remote Sens. 71, 1143-1152 (2005)

F. Scholten, J. Oberst, K.-D. Matz, T. Roatsch, M. Wählisch, M.S. Robinson (The LROC Team), Towards global lunar topography using LROC WAC stereo data, in 41 st Lunar and Planetary Science Conference, vol. 2051 (2009)

H. Sierks, H.U. Keller, R. Jaumann, H. Michalik, F. Bubenhagen, I. Büttner, U. Carsenty, U. Christensen, R. Enge, F. Fiethe, P. Gutiérrez Marqués, H. Hartwig, H. Krüger, W. Kühne, T. Maue, S. Mottola, A. Nathues, K.-U. Reiche, M.L. Richards, T. Roatsch, S.E. Schröder, I. Szemerey, M. Tschentscher, The Dawn framing camera. Space Sci. Rev. (2011, this issue). doi:10.1007/s11214-011-9745-4

P.C. Thomas, R.R. Benzel, M.J. Gaffey, B.H. Zellner, A.D. Storrs, E. Wells, Vesta: spin pole, size, and shape from HST images. Icarus 128, 88-94 (1997) 
P.C. Thomas, J.Wm. Parker, L.A. McFadden, C.T. Russell, S.A. Stern, M.V. Sykes, E.F. Young, Differentiation of the asteroid Ceres as revealed by its shape. Nature 437, 224-226 (2005)

F. Wewel, F. Scholten, K. Gwinner, High resolution stereo camera (HRSC)—multispectral 3D-data acquisition and photogrammetric processing. Can. J. Remote Sens. 26, 466-474 (2000)

M.T. Zuber, D.E. Smith, A.F. Cheng, J.B. Garvin, O. Aharonson, T.D. Cole, P.J. Dunn, Y. Guo, F.G. Lemoine, G.A. Neumann, D.D. Rowlands, M.H. Torrence, The shape of 433 Eros from the NEAR-shoemaker laser rangefinder. Science 289, 2097-2101 (2000). doi:10.1126/science.289.5487.2097 